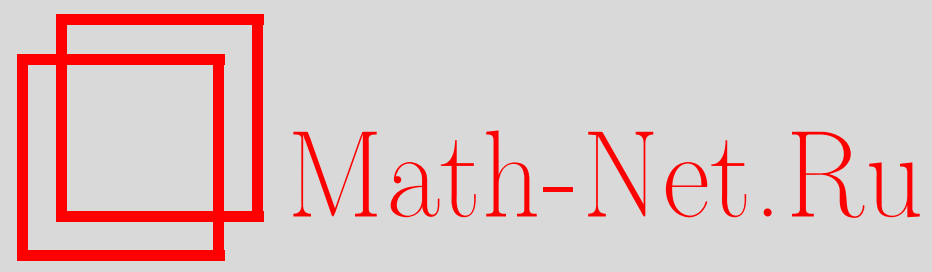

П. Г. Григорьев, Случайные линейные комбинации функций из $L_{1}$, Матем. заметки, 2003, том 74, выпуск 2, $192-$ 220

DOI: https://doi.org/10.4213/mzm257

Использование Общероссийского математического портала Math-Net.Ru подразумевает, что вы прочитали и согласны с пользовательским соглашением http://www . mathnet.ru/rus/agreement

Параметры загрузки:

IP: 35.174 .16 .151

26 апреля 2023 г., 12:38:17

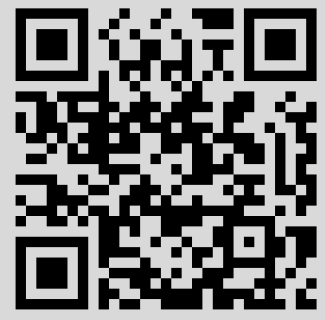


УДК 519.222

\section{СЛУЧАЙНЫЕ ЛИНЕЙНЫЕ КОМБИНАЦИИ ФУНКЦИЙ ИЗ $L_{1}$}

\section{П. Г. Григорьев}

Работа посвящена исследованию свойств полиномов со случайными коэффициентами по общим системам суммируемых функций. Получены нижние оценки математического ожидания равномерной и так назьваемой интегрально-равномерной нормы случайного полинома.

Библиография: 25 названий.

\section{1. Введение}

Статья посвящена исследованию случайных полиномов (линейных комбинаций со случайными коэффициентами) следующего вида:

$$
F_{n}(\omega, x):=\sum_{i=1}^{n} \xi_{i}(\omega) f_{i}(x)
$$

где $\left\{f_{i}\right\}_{1}^{n}$-некоторая система функций на пространстве с мерой $(X, \mu),\left\{\xi_{i}\right\}_{1}^{n}$-набор независимых случайных величин на вероятностном пространстве $(\Omega, \mathrm{P})$ ("случайные коэффициенты”). Нас будут интересовать свойства случайной величины $\left\|F_{n}(\omega, \cdot)\right\|_{B(X)}$, где $\|\cdot\|_{B}$ - норма в некотором пространстве $B$ функций на $(X, \mu)$.

Заметим, что при $B=L_{\infty}$ сформулированная вьше задача преврашается в классическую задачу оценки математического ожидания супремума случайного полинома вида (1). Салемом и Зигмундом [1] в 1954 г. было доказано, что

$$
\mathrm{E}\left\|\sum_{k=-n}^{n} r_{k}(\omega) e^{i k x}\right\|_{\infty} \equiv \frac{1}{2^{2 n+1}} \sum_{\substack{\text { по всем } \\ \text { знакам } \varepsilon_{k}= \pm 1}}\left\|\sum_{k=-n}^{n} \varepsilon_{k} e^{i k x}\right\|_{\infty} \asymp \sqrt{n \log n},
$$

где $r_{k}(\omega)=\operatorname{sign} \sin \left(2^{k+1} \pi \omega\right)$ - функции Радемахера на [0, 1], E- оператор математического ожидания. В то же время из неравенства Хинчина вытекает, что

$$
\mathrm{E}\left\|\sum_{k=-n}^{n} r_{k}(\omega) e^{i k x}\right\|_{p} \asymp_{p} \sqrt{n}, \quad 1 \leqslant p<\infty .
$$

Этот факт показьвает качественное различие вложений пространства тригонометрических полиномов порядка не вьше $n$ в $L_{\infty}$ и в $L_{p}$ при $p<\infty$.

Работа поддержана грантом НШ-№ 1549.2003 .1 и Российским фондом фундаментальных исследований, грант № 02-01-00315. 
K настоящему моменту получен ряд оценок равномерной нормы случайных полиномов вида (1) с различными ограничениями на $\left\{\xi_{i}\right\}_{1}^{n}$ и $\left\{f_{i}\right\}_{1}^{n}$. Отметим, что верхние оценки давно нашли разнообразные приложения в анализе и теории вероятностей, приложения нижних оценок далеко не так многочисленны. Однако именно доказательство нижних оценок наиболее трудоемко и обычно требует привлечения специфических свойств систем $\left\{f_{i}\right\}$ и $\left\{\xi_{i}\right\}$. Так, для доказательства нижней оценки в соотношении (2) в [1] фактически было использовано то, что тригонометрическая система является системой характеров (т.е. использовались тригонометрические формулы типа $\cos (\alpha+\beta)=\cos \alpha \cdot \cos \beta-\sin \alpha \cdot \sin \beta)$, а также экспоненциальная оценка для функций Радемахера. В [2] приведено короткое доказательство нижней оценки равномерной нормы случайного полинома $\sum_{k} \xi_{k} \cos k x$, где $\left\{\xi_{k}\right\}$ - независимые гауссовские величины, которое использует то, что матрица

$$
\left(\cos \frac{2 \pi}{n} j k\right)_{j, k=0}^{n-1}
$$

кратна ортогональной, и то, что некоррелированные гауссовские величины независимы. В доказательстве верхних оценок условия на систему $\left\{f_{i}\right\}$ как правило существенно слабее. Так, обычно, если для математического ожидания нормы полинома вида (1) по системе $\left\{f_{i}\right\}_{1}^{n}$ вьполнена некоторая верхняя оценка, то она имеет место и в том случае, если мы заменим в выражении (1) функции $f_{i}$ их абсолютньми величинами $\left|f_{i}\right|$. В настоящей работе, продолжающей работу [3], получена нижняя оценка равномерной нормы случайных полиномов (1) по абстрактной системе функций $\left\{f_{i}\right\}_{1}^{n}$ из $L_{1}$.

В монографиях [4], [5] излагается обширная теория, позволяюшая получать оценки математического ожидания равномерной нормы случайного полинома (1) в том случае, когда система $\left\{f_{i}\right\}$ является системой характеров на локально-компактной абелевой группе, ограниченной на некоторую компактную окрестность $V$ единичного элемента этой групы. Основным методом получения оценок максимума случайного процесса (в частности, случайного полинома вида (1)) в [4], [5] служит сведение задачи к оценке $\varepsilon$-энтропии $V$ относительно некоторой метрики, индуцируемой этим случайньм процессом. Этот метод был впервые использован Дадли [6] и Судаковым [7], [8] и позднее развит Ферником, Маркусом, Пизье, Талаграном и другими авторами (см. подробнее [4], [5], [9], [10]. Для доказательства нижних оценок максимума случайного процесса этим методом ключевое значение имеет лемма Слепяна [11] для гауссовских векторов. Эта лемма позволяет оценивать снизу вероятность

$$
\mathrm{P}\left\{\max _{1 \leqslant k \leqslant m} \sum_{i=1}^{n} \xi_{i} f_{i}\left(x_{k}\right) \geqslant \alpha\right\}, \quad\left\{x_{k}\right\}_{1}^{m} \text {-некоторая сетка в } X,
$$

в том случае, когда все нормированные скалярные произведения (косинусы углов)

$$
\frac{\left(W_{x_{j}}, W_{x_{k}}\right)}{\left|W_{x_{j}}\right| \cdot\left|W_{x_{k}}\right|}, j \neq k, \text { для векторов } W_{x_{j}}:=\left(f_{i}\left(x_{j}\right)\right)_{i=1}^{n} \in \mathbb{C}^{n}, j=1, \ldots, m,
$$

достаточно малы, а $\left\{\xi_{i}\right\}$ - независимые стандартные гауссовские величины. Доказательство существования такой сетки $\left\{x_{j}\right\}$ представляет в ряде случаев отдельную нетривиальную задачу. Кроме того, поскольку лемма Слепяна применима лишь к гауссовским векторам, возникают серьезные трудности с перенесением оценок для случайных полиномов с гауссовскими коэффишиентами на случай негауссовских $\left\{\xi_{i}\right\}$. 
В 1995 г. Кашиным и Цафрири в работах [12]-[14] был предложен другой метод получения нижних оценок равномерной нормы случайных полиномов. В частности, в [12] было показано, что нижняя оценка в теореме Салема-Зигмунда (2) остается справедливой для случайных полиномов по любой ортонормированной равномерно ограниченной в $L_{3}$ системе. Этот метод опирается на версию двумерной центральной предельной теоремы с оценкой остаточного члена. При этом уже не требуется оценивать все попарные углы между векторами $W_{x_{j}}$, а достаточно показать, что косинусы этих углов $⿻ а л ь \boldsymbol{s}$ среднем.

В [13] Кашин и Цафрири ввели следующую норму:

$$
\|f\|_{m, \infty}:=\int_{X} \cdots \int_{X} \max \left\{\left|f\left(x_{1}\right)\right|, \ldots,\left|f\left(x_{m}\right)\right|\right\} d \mu\left(x_{1}\right) \cdots d \mu\left(x_{m}\right)
$$

где $f$ - интегрируемая функция на пространстве с мерой $(X, \mu), \mu(X)=1$. Следуя работам автора [15], [3], будем назьвать норму $\|f\|_{m, \infty}$ интегрально-равномерной нормой. При доказательстве нижних оценок математического ожидания равномерной нормы случайного полинома (1) в [12] и [14] фактически были получены аналогичные оценки для интегрально-равномерной нормы со значением параметра $m \asymp n^{1 / 2+\varepsilon}$. Стоит заметить, что оценки для $\|f\|_{m, \infty}$ (точнее для семейства норм с $m \geqslant 1$ ) представляют самостоятельньй интерес, так как несут в себе довольно полную информацию о распределении функции $f: \lambda_{f}(t)=\mu\{x \in X:|f(x)| \geqslant t\}$. Так, нетрудно показать, что

$$
\|f\|_{m, \infty}=\int_{0}^{\infty}\left(1-\left(1-\lambda_{f}(t)\right)^{m}\right) d t
$$

и $\|f\|_{1} \equiv\|f\|_{1, \infty} \leqslant\|f\|_{m, \infty} \leqslant\|f\|_{\infty}$, причем $\|f\|_{m, \infty} \rightarrow\|f\|_{\infty}$ при $m \rightarrow \infty$. В разделе 2 (теорема 2) будет показано, что

$$
\|f\|_{m, \infty} \asymp \sup _{\substack{\Delta \subset X \\ \mu \Delta=1 / m}}\left\{m \int_{\Delta}|f| d \mu\right\}
$$

если при $m=1,2, \ldots$ в $X$ существует подмножество меры $1 / m$.

Автором в [3] с помощью несложной модернизации метода, предложенного в [12], [14], была доказана следующая

Теорема А. Для произвольных значений параметров $p \in[0,1 / 2), R, M, \varepsilon>0$ существуют полохсительные постоянные $q=q(p), C_{j}=C_{j}(p, M, \varepsilon), j=1,2,3$, такие, что для произвольных наборов функиий $\left\{f_{i}\right\}_{i=1}^{n}$ и случайньх величин $\left\{\xi_{i}\right\}_{i=1}^{n}$, определенных на вероятностных пространствах $(X, \mu) u(\Omega, \mathrm{P})$ соответственно, и комплексных коэффициентов $\left\{a_{i}\right\}_{i=1}^{n}$, удовлетворяюших условиям

(a) $\left\|f_{i}\right\|_{2}=1 u\left\|f_{i}\right\|_{2+\varepsilon} \leqslant M$ для всех $i=1, \ldots, n$;

(b) $\left\|\sum_{i=1}^{n} c_{i} f_{i}\right\|_{2} \leqslant M R^{p}\left(\sum_{i=1}^{n}\left|c_{i}\right|^{2}\right)^{1 / 2}$ для всех наборов коэффициентов $\left\{c_{i}\right\}_{1}^{n}$, где $R \equiv R\left(\left\{a_{i}\right\}_{1}^{n}\right):=\left(\sum_{i=1}^{n}\left|a_{i}\right|^{2}\right)^{2} / \sum_{i=1}^{n}\left|a_{i}\right|^{4}$;

(c) $\left\{\xi_{i}\right\}_{1}^{n}$ - независимые случайные величины такие, что $\mathrm{E} \xi_{i}=0, \mathrm{E}\left|\xi_{i}\right|^{2}=1 u$ $\left(\mathrm{E}\left|\xi_{i}\right|^{2+\varepsilon}\right) \leqslant M$ 
справедливо неравенство ${ }^{1}$

$$
\mathrm{P}\left\{\omega \in \Omega:\left\|\sum_{i=1}^{n} a_{i} \xi_{i}(\omega) f_{i}\right\|_{m, \infty} \leqslant C_{1}\left(\sum_{i=1}^{n}\left|a_{i}\right|^{2} \log P\right)^{1 / 2}\right\} \leqslant \frac{C_{2}}{P^{q}},
$$

әде $P=\min (m, R)+1, R=R\left(\left\{a_{i}\right\}\right)$, из условия (b), и, следовательно, ${ }^{2}$

$$
\mathrm{E}\left\|\sum_{i=1}^{n} a_{i} \xi_{i} f_{i}\right\|_{m, \infty} \geqslant C_{3}\left(\sum_{i=1}^{n}\left|a_{i}\right|^{2} \log P\right)^{1 / 2} .
$$

Теорема А является несложным обобщением результатов из [12]-[14], где оценивалась $\|\cdot\|_{\infty}$-норма случайньх полиномов. Там оценки типа $(5),(6)$ для равномерной нормы были получены в случае $p=0$ и было замечено, что при $a_{i} \equiv 1$ их можно обобшить на случай $p \in[0,1 / 2)$, а также отмечено, что установленные оценки имеют место и для интегрально-равномерной нормы случайного полинома с параметром $m \asymp R^{1 / 2}$.

Таким образом, оценка типа (6) может быть неверна только для случайного полинома по системе нормированных в $L_{2}$ функций $\left\{f_{i}\right\}$, существенно отличающейся от ортогональной, такой, например, как последовательность функций, сходящаяся к некоторой фиксированной функции.

Заметим, что в случае $a_{i} \equiv 1\left(R\left(\left\{a_{i}\right\}\right)=n\right)$ с помошью леммы 1 (см. ниже) легко вывести условие (b) для функций $\left\{f_{i}\right\}$ таких, что $\left\|f_{i}\right\|_{2}=1$, из следуюшего условия

$\left(\mathrm{b}^{\prime}\right)\left\|\sum_{i=1}^{n} \varepsilon_{i} f_{i}\right\|_{2} \leqslant M n^{1 / 2+\beta}$ для всех наборов знаков $\varepsilon_{i}= \pm 1$ с некоторыми $\beta \in[0,1 / 2), M>0$.

В [3] было также показано, что при $m \leqslant R$ и некоторых дополнительных ограничениях на $\left\{\xi_{k}\right\}_{1}^{n}$ нижняя оценка (6) точна по порядку (при $\left.n \rightarrow \infty\right)$. Точнее, справедлива следующая

ТЕОРемА В. Пусть $\left\{\xi_{k}\right\}_{1}^{n}$ - набор случайных величин, для которых справедлива әкспоненциальная оценка

$$
\mathrm{P}\left\{\left|\sum_{k=1}^{n} c_{k} \xi_{k}\right|>t\left(\sum_{k=1}^{n} c_{k}^{2}\right)^{1 / 2}\right\} \leqslant C_{4} e^{-t^{2} C_{5}}
$$

для всех наборов коэффициентов $\left\{c_{k}\right\}_{1}^{n}$ с некоторыми константами $C_{4}, C_{5}>0$. Тогда для всех наборов функиий $\left\{f_{k}\right\}_{1}^{n} \subset L_{1}(X, \mu), \mu X=1$, при любом $m \geqslant 1$ имеет место неравенство

$$
\mathrm{E}\left\|\sum_{k=1}^{n} \xi_{k} f_{k}\right\|_{m, \infty} \leqslant C_{6}\left\|\left(\sum_{k=1}^{n}\left|f_{k}\right|^{2}\right)^{1 / 2}\right\|_{m, \infty} \cdot \sqrt{1+\log m}
$$

с некоторой константой $C_{6}\left(C_{4}, C_{5}\right)$.

\footnotetext{
${ }^{1}$ Можно показать, что степенной параметр $q$ можно выбрать произвольно из интервала $(0,(1-2 p) / 2)$, при этом константы $C_{1}, C_{2}, C_{3}$ могут зависеть от выбора $q$. Для случая $q=(1-2 p) / 4$ аккуратное доказательство оценки (5) можно найти в [16].

${ }^{2}$ При малых $m$ или $R$ неравенство (6) доказьвается непосредственно с помощью неравенств Хинчина, Гёльдера и тривиальной оценки $\|\cdot\| m, \infty \geqslant\|\cdot\|_{1}$.
} 
Отметим, что условие равномерной ограниченности функций $\left\{f_{i}\right\}$ в $L_{2+\varepsilon}$, входящее в условия теоремы А, для вьполнения жижних оценок типа (5), (6) выглядит довольно неестественно. Дело здесь, грубо говоря, в том, что равномерная ограниченность системы функций в $L_{2+\varepsilon}$ наряду с ее ортонормируемостью (или условием $\left(\mathrm{b}^{\prime}\right)$ при $\left\|f_{i}\right\|_{2}=1$ ) обеспечивают "достаточно сильное" пересечение "существенных" носителей функций $f_{i}$. Чтобы убедиться, что условие равномерной ограниченности не может быть просто отброшено из условия теоремы $\mathrm{A}$, достаточно рассмотреть пример системы функций $f_{i}:=\sqrt{n} \chi_{i}$, где $\chi_{i}-$ характеристические функции интервалов $((i-1) / n, i / n)$.

Основной целью данной работы является обобщение теоремы А в частном случае $a_{i} \equiv 1$ для случайных полиномов вида (1) по системам функций $\left\{f_{i}\right\}_{1}^{n},\left\|f_{i}\right\|_{1}=1$, вообще говоря, неограниченным в $L_{p}$ при $p>1$. При этом вместо условий $(\mathrm{a}),\left(\mathrm{b}^{\prime}\right)$ требуется выполнение условия

(d) $\left\|f_{i}\right\|_{1}=1$ для всех $i=1, \ldots, n$ и $\left\|\sum_{i=1}^{n} \theta_{i} f_{i}\right\|_{1} \leqslant M n^{1 / 2+p}$ для всех наборов знаков $\left\{\theta_{i}\right\}_{1}^{n}, \theta_{i}= \pm 1$,

с некоторыми постоянными $p \in[0,1 / 12), M>0$. Справедлива следуюшая

ТЕОрема 1. Пусть система функиий $\left\{f_{i}\right\}_{i=1}^{n}$ на вероятностном пространстве $(X, \mu)$ удовлетворяет условию $(\mathrm{d})$, причем $p \in[0,1 / 12)$, а независимье случайные величины $\left\{\xi_{i}\right\}_{i=1}^{n}$, определенные на другом вероятностном пространстве $(\Omega, \mathrm{P})$ таковь, что $\mathrm{E} \xi_{i}=0, \mathrm{E}\left|\xi_{i}\right|^{2}=1 u \mathrm{E}\left|\xi_{i}\right|^{3} \leqslant M^{3}$. Тогда при любом $m \leqslant n$ для случайного полинома (1) имеет место неравенство

$$
\mathrm{P}\left\{\omega \in \Omega:\left\|F_{n}(\omega, x)\right\|_{m, \infty} \leqslant C_{1}^{\prime} \sqrt{n \cdot(1+\log m)}\right\} \leqslant C_{2}^{\prime} m^{-q^{\prime}},
$$

и, следовательно,

$$
\mathrm{E}\left\|\sum_{i=1}^{n} \xi_{i} f_{i}\right\|_{m, \infty} \geqslant C_{3}^{\prime}(n \cdot(1+\log m))^{1 / 2}
$$

с некоторыми константами $q^{\prime}=q^{\prime}(p)>0, C_{j}^{\prime}=C_{j}^{\prime}(p, M)>0, j=1,2,3$, зависящими только от р и $M$.

(В случае малых $m$ неравенство (8) доказьвается непосредственно с помошњю неравенства Хинчина.)

Этот результат является новым не только для интегрально-равномерной нормы, но и для равномерной нормы случайных полиномов вида (1). Справедливо

СлЕДСТВИЕ 1. Пусть функиии $\left\{f_{i}\right\}_{1}^{n}$ и случайные величины $\left\{\xi_{i}\right\}_{1}^{n}$ удовлетворяют условию теоремы 1. Тогда для случайного полинома (1) справедлива оченка

$$
\mathrm{P}\left\{\omega \in \Omega:\left\|F_{n}(\omega, x)\right\|_{\infty} \leqslant C_{1}^{\prime} \sqrt{n \cdot(1+\log n)}\right\} \leqslant C_{2}^{\prime} n^{-q^{\prime}} .
$$

Заметим, что теорема 1 слабее теоремы А в том аспекте, что она не может быть применена к случайньм полиномам вида $\sum_{k} a_{k} \xi_{k}(\omega) f_{k}(x)$ с произвольньми неслучайньми коэффищиентами $\left\{a_{i}\right\}$, а также тем, что требует вьполнения условия $(\mathrm{d})$ с параметром $p<1 / 12$, в то время как в аналогичном условии теоремы А (усл. (b) при $a_{i} \equiv 1$ или ( $\left.\mathrm{b}^{\prime}\right)$ ) достаточно ограничения $p<1 / 2$. Автор считает, что условие $p<1 / 12$ можно ослабить ${ }^{3}$ (см. ниже гипотезу 2 ).

\footnotetext{
${ }^{3}$ Свойство (d) с параметром $p=1 / 2$ очевидно выполнено для всех систем функций $\left\{f_{i}\right\}_{1}^{n}$, $\left\|f_{i}\right\|_{1}=1$. В том числе и для $f_{i} \equiv 1$, для которых, в силу неравенства Хинчина, $L_{\infty}$-норма ограничена сверху по порядку $\sqrt{n}$. Поэтому, пытаться доказать оценки типа $(7),(8)$ можно только для случая $p<1 / 2$.
} 
Дальнейшее изложение организовано следующим образом. В разделе 2 мы докажем несколько вспомогательных результатов, которые в явной или неявной форме были использованы в работах [12], [14], [3]. Эти вспомогательные результаты позволяют легче понять идею метода оценки норм случайных полиномов, развитого в [12], [14], [3], а также получить обобщения теоремы А с конкретными значениями степенного параметра $q$ (см. сноску 1 и замечание 2 ниже). В разделе 3 мы завершим доказательство теоремы 1 . В разделе 4 показано, как с помощью результатов раздела 3 можно частично ответить на вопрос, поставленный Монтгомери-Смитом и Семеновьг в [17], а также сформулированы гипотезы относительно потенциальных обобщений теоремы 1.

Автор выражает особую благодарность своему научному руководителю Б. С. Кашину, под руководством которого были получены результаты настоящей статьи, а также благодарит А. М. Зубкова и Е. М. Семенова за ценные замечания и обсуждения.

\section{2. Вспомогательные результаты}

2.1. Интегрально-равномерная норма. Покажем эквивалентность определений (3) и (4) интегрально-равномерной нормы. Известно, что для любой функции $g \in L_{1}(Y, \nu)$ имеет место $\|g\|_{1}=\int_{0}^{\infty} \lambda_{g}(t) d t$, где $\lambda_{g}(t):=\nu\{y:|g(y)| \geqslant t\}$. Поэтому, чтобы обосновать эквивалентность (3) и (4), достаточно заметить, что функция

$$
g(\bar{x})=\max \left\{\left|f\left(x_{1}\right)\right|, \ldots,\left|f\left(x_{m}\right)\right|\right\}, \quad \bar{x}=\left(x_{j}\right)_{1}^{m} \in X^{m}=: Y
$$

обладает распределением $\lambda_{g}(t)=1-\left(1-\lambda_{f}(t)\right)^{m}$.

$\mathrm{C}$ помошью неравенства $\max (|a|,|b|) \leqslant|a|+|b|$ легко видеть, что

$$
\|f\|_{m, \infty} \leqslant m\|f\|_{1} \quad \text { и }\|f\|_{n, \infty} \leqslant \frac{n+1}{m}\|f\|_{m, \infty} \quad \text { для всех } f \in L_{1}(X), \quad m \leqslant n .
$$

Для индикатора $\chi_{\Delta}$ множества $\Delta \subset X$ тождество (4) влечет $\left\|\chi_{\Delta}\right\|_{m, \infty}=1-(1-|\Delta|)^{m}$ (здесь и далее обозначаем $|\Delta| \equiv \mu \Delta$ ). Таким образом, при $m \geqslant c|\Delta|^{-1}$ величина $\left\|\chi_{\Delta}\right\|_{m, \infty} \geqslant C^{\prime}(c)$ с некоторой константой $C^{\prime}(c)>0$.

Для интегрируемой функции $f \in L_{1}$ определим следуюшие "родственные" нормы:

$$
\begin{aligned}
& \|f\|_{m}^{*}:=\sup _{\Delta \subset X}\left\{\frac{1-(1-|\Delta|)^{m}}{|\Delta|} \int_{\Delta}|f| d \mu\right\}, \\
& \|f\|_{m}^{\prime}:=\sup _{\substack{\Delta \subset X \\
\mu \Delta=1 / m}}\left\{m \int_{\Delta}|f| d \mu\right\} .
\end{aligned}
$$

Правая часть (11) корректно определена, только если существует событие $e \subset X$ такое, что $\mu(e)=1 / m$. Однако мы будем использовать эту норму, только когда $X=[0,1]$ со стандартной мерой Лебега.

Нормы $\|\cdot\|_{m}^{*}$ и $\|\cdot\|_{m}^{\prime}$ эквивалентны $\|\cdot\|_{m, \infty}$-норме, это вытекает из следующего результата, в неявной форме полученного автором в [3].

ТЕОрема 2. Для любой функиии $f \in L_{1}[0,1]$ имеют место неравенства

$$
\left(1-\frac{1}{e}\right)\|f\|_{m}^{\prime} \leqslant\|f\|_{m}^{*} \leqslant\|f\|_{m, \infty} \leqslant 2\|f\|_{m}^{\prime} .
$$


ДокАЗАтЕЛЬСтво. Покажем, что $2\|f\|_{m}^{\prime} \geqslant\|f\|_{m, \infty}$. Пусть множество $\Delta^{*} \subset[0,1]$ таково, что $\left|\Delta^{*}\right|=1 / m$ и

$$
m \int_{\Delta^{*}}|f|=\|f\|_{m}^{\prime}
$$

(Легко видеть, что такое $\Delta^{*}$ сушествует, хотя, может быть, неединственно.) Имеем

$$
\|f\|_{m, \infty} \leqslant\left\|f \cdot \chi_{\Delta^{*}}\right\|_{m, \infty}+\left\|f \cdot\left(1-\chi_{\Delta^{*}}\right)\right\|_{m, \infty}
$$

С помошью (9) оценим $\left\|f \cdot \chi_{\Delta^{*}}\right\|_{m, \infty} \leqslant m \int_{\Delta^{*}}|f|=\|f\|_{m}^{\prime}$. Из экстремальности множества $\Delta^{*}$ получим

$$
\left\|f \cdot\left(1-\chi_{\Delta^{*}}\right)\right\|_{m, \infty} \leqslant\left\|f \cdot\left(1-\chi_{\Delta^{*}}\right)\right\|_{\infty} \leqslant \frac{1}{\left|\Delta^{*}\right|} \int_{\Delta^{*}}|f|=\|f\|_{m}^{\prime} .
$$

Неравенство $\|f\|_{m, \infty} \leqslant 2\|f\|_{m}^{\prime}$ доказано.

Докажем теперь, что для любого $\Delta \subset[0,1]$ имеет место неравенство

$$
\|f\|_{m, \infty} \geqslant \frac{1-(1-|\Delta|)^{m}}{|\Delta|} \int_{\Delta}|f| d \mu .
$$

Очевидно, можно ограничиться случаем, когда носитель supp $f \subset \Delta$. С помошью формулы (4) получим

$$
\begin{aligned}
\|f\|_{k+1, \infty}-\|f\|_{k, \infty} & =\int_{0}^{\infty}\left(1-\left(1-\lambda_{f}(t)\right)^{k+1}\right) d t-\int_{0}^{\infty}\left(1-\left(1-\lambda_{f}(t)\right)^{k}\right) d t \\
& =\int_{0}^{\infty} \lambda_{f}(t)\left(1-\lambda_{f}(t)\right)^{k} d t \\
& \geqslant(1-|\Delta|)^{k} \int_{0}^{\infty} \lambda_{f}(t) d t=(1-|\Delta|)^{k}\|f\|_{1} .
\end{aligned}
$$

Суммируя эти неравенства от $k=1$ до $k=m-1$, получим, что

$$
\|f\|_{m, \infty}-\|f\|_{1} \equiv\|f\|_{m, \infty}-\|f\|_{1, \infty} \geqslant\|f\|_{1} \sum_{k=1}^{m-1}(1-|\Delta|)^{k}
$$

что влечет

$$
\|f\|_{m, \infty} \geqslant\|f\|_{1} \frac{1-(1-|\Delta|)^{m}}{|\Delta|} .
$$

Чтобы доказать (13), нам осталось заметить, что $\|f\|_{1}=\int_{\Delta}|f|($ так как $\operatorname{supp} f \subset \Delta)$. Следовательно, $\|f\|_{m, \infty} \geqslant\|f\|_{m}^{*}$.

Неравенство $\|f\|_{m}^{*} \geqslant\left(1-e^{-1}\right)\|f\|_{m}^{\prime}$ очевидным образом следует из того, что $(1-1 / m)^{m}<e^{-1}$. Теорема 2 доказана. 


\section{2. Геометрический факт.}

ЛЕмма 1 (см. [3, лемма 1] или [4, лемма 2.1]). Пусть $\left\{w_{i}\right\}_{i=1}^{n}-$ набор векторов в линейном пространстве с нормой (или полунормой) $\|\cdot\|$ таких, что $\left\|w_{i}\right\|=1 u$

$$
\left\|\sum_{i=1}^{n} \theta_{i} w_{i}\right\| \leqslant c \cdot n^{1 / 2+\beta}
$$

для всех наборов знаков $\left\{\theta_{i}\right\}_{i=1}^{n}, \theta_{i}= \pm 1$, с некоторыми константами $\beta \in[0,1 / 2)$, $c>0$. Тогда

$$
\left\|\sum_{i=1}^{n} a_{i} w_{i}\right\| \leqslant C(c) n^{1 / 4+\beta / 2}\left(\sum_{i=1}^{n} a_{i}^{2}\right)^{1 / 2}
$$

для всех наборов коэффициентов $\left\{a_{i}\right\}_{1}^{n}$. Эта оиенка неулучиаема в том смысле, что существуют норма $\|\cdot\|$, векторь $\left\{w_{i}\right\}_{1}^{n}$ и коэффичиенты $\left\{a_{i}\right\}_{1}^{n}$ такие, что оценка (14) имеет место, а оченка $\left(14^{\prime}\right)$ точна по порядку (при $\left.n \rightarrow \infty\right)$.

Геометрически лемма 1 утверждает, что радиус вписанного шара выпуклой оболочки множества $B_{\infty}^{d} \cup\left(n^{1 / 2+\beta} \cdot B_{1}^{d}\right)$ равен по порядку $n^{1 / 4+\beta / 2}$, где $B_{\infty}^{d}$ обозначает $d$-мерный куб с вершинами, имеюшими координаты \pm 1 , а $B_{1}^{d}=\left\{\left(v_{k}\right) \in \mathbb{R}^{d}: \sum_{1}^{d}\left|v_{k}\right| \leqslant 1\right\}$.

2.3. Леммы перехода. Для доказательства основного результата нам потребуются следующие леммы, которые позволяют переносить одно свойство многомерного нормального распределения (лемма 3) на случай многомерного распределения общего вида.

ЛЕмма 2. Пусть события $\Omega_{j} \subset \Omega, j=1, \ldots, m$, обладают следующим свойством:

$$
(1-\kappa) \sum_{j, k=1}^{m} \mathrm{P}\left(\Omega_{j} \cap \Omega_{k}\right) \leqslant\left(\sum_{j=1}^{m} \mathrm{P}\left(\Omega_{j}\right)\right)^{2}
$$

с некоторым $\kappa \in(0,1)$. Тогда $\mathrm{P}\left(\bigcup_{j=1}^{m} \Omega_{j}\right) \geqslant 1-\kappa$.

ЛЕмма 3. Пусть $\left\{h_{j}\right\}_{j=1}^{m}$ - набор гауссовских случайных величин таких, что $\mathrm{E} h_{j}=0, \mathrm{E} h_{j}^{2}=D_{j} \geqslant r^{2}>0, \mathrm{E} h_{j} h_{k}=v_{j, k} u$ кажсдая пара $\left(h_{j}, h_{k}\right), j \neq k$, имеет двумерное нормальное распределение с плотностью

$$
\begin{gathered}
\phi_{0, V_{j, k}}(Y):=\frac{1}{2 \pi\left(\operatorname{det} V_{j, k}\right)^{1 / 2}} \exp \left\{-\frac{1}{2}\left(Y, V_{j, k}^{-1} Y\right)\right\}, \quad Y=\left(y_{1}, y_{2}\right), \\
\text { əде } V_{j, k}=\left(\begin{array}{cc}
D_{j} & v_{j, k} \\
v_{j, k} & D_{k}
\end{array}\right)-\text { ковариационная матрица. }
\end{gathered}
$$

Пусть также существуют константы $R \geqslant 1, c_{0}, \delta>0$ такие, что

$$
\frac{1}{m^{2}} \sum_{\substack{j, k=1 \\ j \neq k}}^{m}\left|v_{j, k}\right| \leqslant c_{0} R^{-\delta} r^{2}
$$

Тогда для любого $\alpha<\delta^{1 / 2}$ существует не зависящая от $R$ константа $C_{7}=$ $C_{7}\left(c_{0}, \alpha, \delta\right)$ такая, что для событий

$$
\Psi_{j}=\Psi_{j}(\alpha):=\left\{h_{j}>\alpha \sqrt{D_{j} \log P}\right\}, \quad \text { əде } \quad P:=\min (R, m)+1,
$$


справедливо неравенство

$$
\sum_{j, k=1}^{m} \mathrm{P}\left(\Psi_{j} \cap \Psi_{k}\right) \leqslant\left(1+C_{7} P^{-q_{0}}\right)\left(\sum_{j=1}^{m} \mathrm{P}\left(\Psi_{j}\right)\right)^{2},
$$

əде $q_{0}=q_{0}(\alpha, \delta)=\min \left(\left(\delta-\alpha^{2}\right) / 3,3 \alpha^{2} / 2\right)>0$.

Лемма 4. Пусть $\left(\eta_{j}\right)_{1}^{m} u\left(h_{j}\right)_{1}^{m}$ - случайные векторы такие, что

$$
\mathrm{E} \eta_{j}=\mathrm{E} h_{j}=0, \quad \mathrm{E} \eta_{j} \eta_{k}=\mathrm{E} h_{j} h_{k}=v_{j, k}, \quad D_{j} \equiv v_{j, j} \geqslant r^{2}>0,
$$

причем вектор $\left(h_{j}\right)_{1}^{m}$ гауссовский, ковариационная матрица которого удовлетворяет условиям леммы 3 с параметрами $r, R, c_{0}, \delta>0$. Предположим также, что для некоторых полохительных постоянных $\delta_{i} \leqslant 1, M_{i}, i=1,2,3, u$ $\alpha<\alpha_{0}:=\left(\min \left(\delta, \delta_{1}, \delta_{2}, \delta_{3}\right)\right)^{1 / 2}$ выполнены следующие условия:

$$
\begin{gathered}
\left|\mathrm{P}\left(U_{j}(\alpha)\right)-\mathrm{P}\left(\Psi_{j}(\alpha)\right)\right| \leqslant M_{1} P^{-\delta_{1}}, \quad 1 \leqslant j \leqslant n, \\
\left|\mathrm{P}\left(U_{j}(\alpha) \cap U_{k}(\alpha)\right)-\mathrm{P}\left(\Psi_{j}(\alpha) \cap \Psi_{k}(\alpha)\right)\right| \leqslant M_{2} P^{-\delta_{2}}, \quad(j, k) \in \sigma,
\end{gathered}
$$

əде $P=\min (R, m)+1 u$

$$
\begin{gathered}
\Psi_{j}=\Psi(\alpha):=\left\{\omega: h_{j}(\omega)>\alpha \sqrt{D_{j} \log P}\right\}, \\
U_{j}=U_{j}(\alpha):=\left\{\omega: \eta_{j}(\omega)>\alpha \sqrt{D_{j} \log P}\right\},
\end{gathered}
$$

а множество индексов

$$
\sigma \subset\{(j, k): j \neq k, 1 \leqslant j, k \leqslant m\}
$$

maкое, ито $|\sigma| \geqslant m^{2}\left(1-M_{3} P^{-\delta_{3}}\right)$.

Тогда существует константа $C_{7}^{\prime}=C_{7}^{\prime}\left(\alpha, c_{0}, \delta, \delta_{i}, M_{i}\right), i=1,2,3$, такая, что

$$
\sum_{j, k=1}^{m} \mathrm{P}\left(U_{j}(\alpha) \cap U_{k}(\alpha)\right) \leqslant\left(1+C_{7}^{\prime} P^{-q^{\prime}}\right)\left(\sum_{j=1}^{m} \mathrm{P}\left(U_{j}(\alpha)\right)\right)^{2},
$$

әде степенной параметр

$$
q^{\prime}=\min \left\{\frac{1}{3}\left(\delta-\alpha^{2}\right), \frac{3}{2} \alpha^{2}, \frac{5}{6} \min _{i=1,2,3}\left(\delta_{i}-\alpha^{2}\right)\right\}>0 .
$$

ЗАмЕЧАниЕ 1 (к леммам 2-4). Лемма 2, являющаяся обобщением леммы БореляКантелли, - известньй и важньй результат. Подобные утверждения использовались, например, в [18], [19], [12]. Леммы 3,4 , насколько нам известно, формулируются впервые, однако идея их доказательства полностью позаимствована из [12], [14]. Лемма 4 служит для переноса оценки типа (15) на "негауссовский случай." Отметим, что основные результаты работ [12], [14], [3] проще получить с помошью лемм 2 и 4.

Заметим также, что из лемм 2 и 3 легко вьвести, что для гауссовских случайных величин $\left\{h_{i}\right\}_{1}^{m}$ из формулировки леммы 3 имеет место оценка

$$
\mathrm{P}\left(\max _{1 \leqslant j \leqslant m} h_{j}>\alpha r \sqrt{\log m}\right) \geqslant 1-C m^{-q_{0}} \quad \text { при } m \leqslant R
$$

( $R$ и $r$ из формулировки леммы 3 ) с некоторыми положительными константами $\alpha, q_{0}, C$. Этот факт объединяет лемму 3 с результатами Слепяна [11] и Шидака [20], [21] о распределении максимума гауссовских векторов с нетривиальной ковариационной матрицей. 
ДОКАЗАТЕЛЬСТВО ЛЕММЫ 2. Пусть $\chi_{j}-$ индикаторы событий $\Omega_{j} \subset \Omega$, и пусть $\zeta:=\sum_{j=1}^{n} \chi_{j}$. Тогда $\mathrm{E}|\zeta|=\sum_{j=1}^{m} \mathrm{P}\left(\Omega_{j}\right)$ и $\mathrm{E}|\zeta|^{2}=\sum_{j, k=1}^{m} \mathrm{P}\left(\Omega_{j} \cap \Omega_{k}\right)$. Очевидно, что $\operatorname{supp} \zeta=\bigcup_{j=1}^{m} \Omega_{j}$. С помошью неравенства Коши-Шварца получим

$$
(1-\kappa)^{1 / 2}\left(\mathrm{E}|\zeta|^{2}\right)^{1 / 2} \leqslant \mathrm{E}|\zeta| \leqslant\left(\mathrm{E}|\zeta|^{2}\right)^{1 / 2}\left(\mathrm{P}\left(\bigcup_{j=1}^{m} \Omega_{j}\right)\right)^{1 / 2}
$$

Лемма 2 доказана.

ДокАЗАТЕЛЬСТВо ЛЕмМы 3 . Так как случайные величины $h_{j}$ нормальны, имеем

$$
\mathrm{P}\left(\Psi_{j}\right)=\frac{1}{\sqrt{2 \pi D_{j}}} \int_{\alpha \sqrt{D_{j} \log P}}^{\infty} e^{-y^{2} /\left(2 D_{j}\right)} d y=\frac{1}{\sqrt{2 \pi}} \int_{\alpha \sqrt{\log P}}^{\infty} e^{-y^{2} / 2} d y .
$$

Учитьвая, что $\int_{z}^{\infty} e^{-t^{2} / 2} d t \asymp z^{-1} e^{-z^{2} / 2}$ при $z \geqslant 1$, имеем

$$
C_{\alpha}^{-1}(\sqrt{\log P})^{-1} P^{-\alpha^{2} / 2} \leqslant \mathrm{P}\left(\Psi_{j}\right) \leqslant C_{\alpha}(\sqrt{\log P})^{-1} P^{-\alpha^{2} / 2}
$$

с некоторой константой $C_{\alpha}$, зависящей только от $\alpha$. Следовательно,

$$
\sum_{j=1}^{m} \mathrm{P}\left(\Psi_{j}\right) \asymp \alpha \frac{m}{\alpha \sqrt{\log P}} P^{-\alpha^{2} / 2} .
$$

Поэтому, чтобы доказать (15), нам достаточно проверить, что

$$
\sum_{\substack{j, k=1 \\ j \neq k}}^{m} \mathrm{P}\left(\Psi_{j} \cap \Psi_{k}\right) \leqslant\left(1+C_{7}^{\prime} P^{-q_{0}}\right)\left(\sum_{j=1}^{m} \mathrm{P}\left(\Psi_{j}\right)\right)^{2}
$$

Определим следующее множество индексов:

$$
\sigma_{1}:=\left\{(j, k): 1 \leqslant j \neq k \leqslant m,\left|v_{j, k}\right|<\frac{1}{32} r^{2}\right\} .
$$

Неравенство Чебьшёва для множества $\sigma_{1}^{c}=\{(j, k): 1 \leqslant j \neq k \leqslant m\} \backslash \sigma_{1}$ дает

$$
\left|\sigma_{1}^{c}\right| \leqslant \frac{32}{r^{2}} \sum_{j \neq k}\left|v_{j, k}\right| \leqslant 32 c_{0} m^{2} R^{-\delta}
$$

Следовательно,

$$
\sum_{(j, k) \in \sigma_{1}^{c}} \mathrm{P}\left(\Psi_{j} \cap \Psi_{k}\right) \leqslant 32 c_{0} m^{2} R^{-\delta} \max _{1 \leqslant j \leqslant m} \mathrm{P}\left(\Psi_{j}\right) .
$$

С помощью (19) получим

$$
\sum_{(j, k) \in \sigma_{1}^{c}} \mathrm{P}\left(\Psi_{j} \cap \Psi_{k}\right) \leqslant 32 c_{0} m^{2} R^{-\delta} \frac{\text { const }}{\alpha \sqrt{\log P}} P^{-\alpha^{2} / 2} .
$$


Учитьвая (20), имеем

$$
\sum_{(j, k) \in \sigma_{1}^{c}} \mathrm{P}\left(\Psi_{j} \cap \Psi_{k}\right) \leqslant K_{2} R^{-\delta+\alpha^{2}} \cdot\left(\sum_{j=1}^{m} \mathrm{P}\left(\Psi_{j}\right)\right)^{2}
$$

с некоторой константой $K_{2}\left(\alpha, c_{0}\right)>0$ и, следовательно, при доказательстве $(21)$ суммирование по $\sigma_{1}^{c}$ можно не учитывать.

Оценим теперь сумму

$$
\begin{aligned}
\Sigma_{1} & :=\sum_{s=(j, k) \in \sigma_{1}} \mathrm{P}\left(\Psi_{j} \cap \Psi_{k}\right) \\
& =\int_{\alpha \sqrt{D_{j} \log P}}^{\infty} \int_{\alpha \sqrt{D_{k} \log P}}^{\infty} \sum_{s \in \sigma_{1}} \frac{\exp \left\{-\left(Y, V_{s}^{-1} Y\right) / 2\right\}}{2 \pi \sqrt{\operatorname{det} V_{s}}} d y_{1} d y_{2} .
\end{aligned}
$$

С помощью замены переменных $t_{1}=y_{1} / \sqrt{D_{j}}, t_{2}=y_{2} / \sqrt{D_{k}}$, получим

$$
\Sigma_{1}=\int_{\alpha \sqrt{\log P}}^{\infty} \int_{\alpha \sqrt{\log P}}^{\infty} \sum_{s \in \sigma_{1}} \frac{\sqrt{D_{j} D_{k}}}{2 \pi \sqrt{\operatorname{det} V_{s}}} e^{-Q\left(t_{1}, t_{2}\right) / 2} d t_{1} d t_{2},
$$

где $Q\left(t_{1}, t_{2}\right):=\left(Y, V_{s}^{-1} Y\right)-$ квадратичная форма. Заметим, что определитель $\operatorname{det} V_{s}=$ $D_{j} D_{k}-v_{s}^{2}$ и

$$
V_{s}^{-1}=\frac{1}{D_{j} D_{k}-v_{s}^{2}}\left(\begin{array}{cc}
D_{k} & -v_{s} \\
-v_{s} & D_{j}
\end{array}\right) .
$$

Поэтому коэффициенты квадратичной формы $Q=a_{s} t_{1}^{2}+a_{s} t_{2}^{2}-2 b_{s} t_{1} t_{2}$ определяются следуюшим образом:

$$
a_{s}=\frac{D_{j} D_{k}}{D_{j} D_{k}-v_{s}^{2}}, \quad b_{s}=\frac{v_{s} \sqrt{D_{j} D_{k}}}{D_{j} D_{k}-v_{s}^{2}} .
$$

Заметим также, что $\left|v_{s}\right| \leqslant r^{2} / 32$ при $s \in \sigma_{1}$. Поэтому, принимая во внимание, что $D_{j} \geqslant r^{2}$ при всех $j=1, \ldots, m$, получим

$$
a_{s}-b_{s}=\frac{\sqrt{D_{j} D_{k}}}{\sqrt{D_{j} D_{k}}+v_{s}} \geqslant \frac{1}{2} .
$$

Теперь мы можем оценить значения квадратичной формы $Q$ :

$$
Q\left(t_{1}, t_{2}\right)=a_{s} t_{1}^{2}+a_{s} t_{2}^{2}-2 b_{s} t_{1} t_{2} \geqslant\left(a_{s}-b_{s}\right)\left(t_{1}^{2}+t_{2}^{2}\right) \geqslant \frac{1}{2}\left(t_{1}^{2}+t_{2}^{2}\right) .
$$

Далее, для любого $L>1$ имеем

$$
\begin{aligned}
J(L) & :=\frac{1}{2 \pi} \int_{L \alpha \sqrt{\log P}}^{\infty} \int_{\alpha \sqrt{\log P}}^{\infty} \sum_{s \in \sigma_{1}}\left(\frac{D_{j} D_{k}}{D_{j} D_{k}-v_{s}^{2}}\right)^{1 / 2} e^{-Q\left(t_{1}, t_{2}\right) / 2} d t_{1} d t_{2} \\
& \leqslant \int_{L \alpha \sqrt{\log P}}^{\infty} \int_{\alpha \sqrt{\log P}}^{\infty} \sum_{s \in \sigma_{1}} e^{-\left(t_{1}^{2}+t_{2}^{2}\right) / 4} d t_{1} d t_{2} \\
& \asymp \alpha \frac{\left|\sigma_{1}\right|}{L \log P} e^{-\alpha^{2} / 4\left(L^{2}+1\right) \log P} \leqslant \frac{K_{3} m^{2}}{L \log P} P^{-\alpha^{2}\left(L^{2}+1\right) / 4}
\end{aligned}
$$


с некоторой константой $K_{3}(\alpha)$. Выбрав $L=3$, учитьвая $(20)$, получим, что

$$
J(3)<K_{4} P^{-3 \alpha^{2} / 2}\left(\sum_{j=1}^{m} \mathrm{P}\left(\Psi_{j}\right)\right)^{2}
$$

с некоторой константой $K_{4}=K_{4}(\alpha)>0$.

Заметим, что

$$
\Sigma_{1} \leqslant 2 J(3)+\frac{1}{2 \pi} \int_{\alpha \sqrt{\log P}}^{3 \alpha \sqrt{\log P}} \int_{\alpha \sqrt{\log P}}^{3 \alpha \sqrt{\log P}} \sum_{s \in \sigma_{1}} \sqrt{a_{s}} \cdot e^{-Q\left(t_{1}, t_{2}\right) / 2} d t_{1} d t_{2} .
$$

Теперь, чтобы доказать (15), нам осталось сравнить выражение

$$
A:=\frac{1}{2 \pi} \sum_{s \in \sigma_{1}} \sqrt{a_{s}} e^{-Q\left(t_{1}, t_{2}\right) / 2}
$$

с выражением

$$
B:=\frac{1}{2 \pi}\left|\sigma_{1}\right| \exp \left\{-\frac{1}{2}\left(t_{1}^{2}+t_{2}^{2}\right)\right\}
$$

на квадрате $\alpha \sqrt{\log P} \leqslant t_{1}, t_{2} \leqslant 3 \alpha \sqrt{\log P}$. Действительно, если мы покажем, что $A \leqslant B\left(1+K_{5} P^{-q_{0}}\right)$ с некоторой константой $K_{5}(\alpha, \delta)>0$, то, проинтегрировав это неравенство, получим

$$
\begin{aligned}
\frac{1}{2 \pi} & \int_{\alpha \sqrt{\log P}}^{3 \alpha \sqrt{\log P}} \int_{\alpha \sqrt{\log P}}^{3 \alpha \sqrt{\log P}} \sum_{s \in \sigma_{1}} \sqrt{a_{s}} \exp \left\{-\frac{1}{2} Q\left(t_{1}, t_{2}\right)\right\} d t_{1} d t_{2} \\
& <\left(1+K_{5} P^{-q_{0}}\right) \int_{\alpha \sqrt{\log P}}^{\infty} \int_{\alpha \sqrt{\log P}}^{\infty} \frac{\left|\sigma_{1}\right|}{2 \pi} \exp \left\{-\frac{1}{2}\left(t_{1}^{2}+t_{2}^{2}\right)\right\} d t_{1} d t_{2} \\
& =\left(1+K_{5} P^{-q_{0}}\right) \sum_{s \in \sigma_{1}} \mathrm{P}\left(\Psi_{j}\right) \cdot \mathrm{P}\left(\Psi_{k}\right)
\end{aligned}
$$

что с учетом (22) и (23) влечет (15).

Разобьем множество индексов $\sigma_{1}$ на подмножества

$$
\sigma_{i}:=\left\{s \in \sigma_{1}: 2^{-i} r^{2} \leqslant\left|v_{s}\right|<2^{-i+1} r^{2}\right\}, \quad i=6,7, \ldots
$$

Легко видеть, что $\sigma_{1}=\bigcup_{i \geqslant 6} \sigma_{i}$. С помошью неравенства Чебышёва для $\left|\sigma_{i}\right|$ имеем

$$
\left|\sigma_{i}\right| 2^{-i} r^{2} \leqslant \sum_{s \in \sigma_{1}}\left|v_{s}\right| \leqslant c_{0} r^{2} m^{2} R^{-\delta}
$$

и, следовательно,

$$
\left|\sigma_{i}\right| \leqslant \min \left(m^{2}, 2^{i} c_{0} m^{2} R^{-\delta}\right) .
$$

Учитьвая, что $D_{j} \geqslant r^{2}$, для $s \in \sigma_{i}$ имеем

$$
\begin{aligned}
& \left|\left(a_{s}-1\right)\left(t_{1}^{2}+t_{2}^{2}\right)-2 b_{s} t_{1} t_{2}\right| \leqslant\left(\left|a_{s}-1\right|+\left|b_{s}\right|\right)\left(t_{1}^{2}+t_{2}^{2}\right) \\
& \quad=\frac{\left|v_{s}\right|}{\sqrt{D_{j} D_{k}}-\left|v_{s}\right|}\left(t_{1}^{2}+t_{2}^{2}\right)<2^{2-i}\left(t_{1}^{2}+t_{2}^{2}\right) \leqslant 2^{7-i} \alpha^{2} \log P
\end{aligned}
$$


в области $\alpha \sqrt{\log P} \leqslant t_{1}, t_{2} \leqslant 3 \alpha \sqrt{\log P}$. Кроме того, легко видеть, что для $s \in \sigma_{i}, i \geqslant 6$, выполнено

$$
\sqrt{a_{s}}=\left(\frac{D_{j} D_{k}}{D_{j} D_{k}-v_{s}^{2}}\right)^{1 / 2}=\left(1+\frac{v_{s}^{2}}{D_{j} D_{k}-v_{s}^{2}}\right)^{1 / 2} \leqslant 1+2^{3-2 i} .
$$

Собирая все вместе, получим

$$
A \leqslant \frac{B}{\left|\sigma_{1}\right|} S
$$

где $S:=\sum_{i=6}^{\infty}\left|\sigma_{i}\right|\left(1+2^{3-2 i}\right) e^{\left(2^{7-i} \alpha^{2} \log P\right) / 2}=\sum_{i=6}^{\left[2 q_{0} \log _{2} P\right]}+\sum_{i>\left[2 q_{0} \log _{2} P\right]}^{\infty}=: S_{1}+S_{2}$.

Учитьвая, что $q_{0}=\min \left(\left(\delta-\alpha^{2}\right) / 3,3 \alpha^{2} / 2\right)$, имеем

$$
S_{1} \leqslant \sum_{i=6}^{\left[2 q_{0} \log _{2} P\right]} 2^{i} c_{0} m^{2} R^{-\delta} \cdot\left(1+2^{-9}\right) P^{\alpha^{2}} \leqslant 4 c_{0} m^{2} P^{\alpha^{2}+2 q_{0}} R^{-\delta} \leqslant K_{6} R^{-q_{0}}\left|\sigma_{1}\right|
$$

с некоторой постоянной $K_{6}=K_{6}\left(c_{0}\right)>0$.

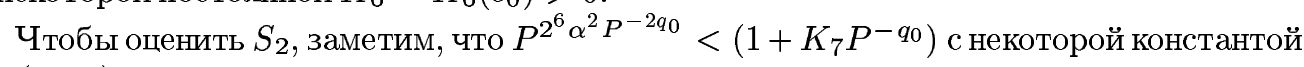
$K_{7}\left(\alpha, q_{0}\right)>0$. Поэтому

$$
S_{2} \leqslant \sum_{i>\left[2 q_{0} \log _{2} P\right]}\left|\sigma_{i}\right|\left(1+8 P^{-4 q_{0}}\right)\left(1+K_{7} P^{-q_{0}}\right) \leqslant\left|\sigma_{1}\right|\left(1+K_{8} P^{-q_{0}}\right)
$$

с константой $K_{8}=8+9 K_{7}$. Следовательно,

$$
S \leqslant\left(1+\left(K_{6}+K_{8}\right) P^{-q_{0}}\right)\left|\sigma_{1}\right|
$$

и $A \leqslant B\left(1+K_{5} P^{-q_{0}}\right)$. Лемма 3 доказана.

ЗАмЕчАниЕ 2. В утверждении леммы степенной показатель $q_{0}(\alpha, \delta)$ выбран неоптимальньм образом. Несложно показать, что неравенство (15) вьполнено и при любом $q_{0}=q_{0}(\alpha, \delta, \varepsilon)=\min \left\{\left(\delta-\alpha^{2}\right) / 2,3 \alpha^{2} / 2\right\}-\varepsilon>0$. (Чтобы увидеть это, достаточно чуть лучше оценить сумму $S)$. Однако в этом случае константа $C_{7}$ в неравенстве $(15)$ будет зависеть еще и от $\varepsilon>0$. Несколько более сложная модернизация доказательства позволяет получить (15) с параметром $q_{0}=\left(\delta-\alpha^{2}\right) / 2-\varepsilon$.

ДокАЗАТЕЛЬСТво ЛЕмМЫ 4 . Поскольку для случайного вектора $\left(h_{j}\right)_{1}^{m}$ выполнены условия леммы 3 , для событий $\left\{\Psi_{j}\right\}_{j=1}^{m}$ имеет место оценка (15) со степенным параметром $q_{0}=\left(\delta-\alpha^{2}\right) / 3$. Следовательно, чтобы установить $(18)$, нам достаточно доказать, что для любого $\alpha<\alpha_{0}$ вьполнены неравенства

$$
\begin{gathered}
\sum_{j=1}^{m} \mathrm{P}\left(\Psi_{j}(\alpha)\right) \leqslant\left(1+L_{1} P^{-q_{1}}\right) \sum_{j=1}^{m} \mathrm{P}\left(U_{j}(\alpha)\right) \\
\sum_{j, k=1}^{m} \mathrm{P}\left(U_{j}(\alpha) \cap U_{k}(\alpha)\right) \leqslant \sum_{j, k=1}^{m} \mathrm{P}\left(\Psi_{j}(\alpha) \cap \Psi_{k}(\alpha)\right)+\frac{L_{2}}{P^{q_{2}}}\left(\sum_{j=1}^{m} \mathrm{P}\left(\Psi_{j}(\alpha)\right)\right)^{2}
\end{gathered}
$$

с константами $q_{1}, q_{2} \geqslant q^{\prime}$ и $L_{1}, L_{2}>0$, зависящими только от $\alpha, c_{0}, \delta, \delta_{i}, M_{i}, i=1,2,3$. 
Заметим, что

$$
\mathrm{P}\left(\Psi_{j}(\alpha)\right)=\frac{1}{2 \pi D_{j}} \int_{\alpha \sqrt{D_{j} \log P}}^{\infty} e^{-y^{2} /\left(2 D_{j}\right)} d y \asymp \alpha \frac{P^{-\alpha^{2} / 2}}{\alpha \sqrt{\log P}} .
$$

Поэтому при любом $\alpha^{2}<\delta_{1}$ имеем (см. (16))

$$
\begin{gathered}
\mathrm{P}\left(U_{j}(\alpha)\right) \asymp_{\alpha} \mathrm{P}\left(\Psi_{j}(\alpha)\right), \\
M_{1} P^{-\delta_{1}} \leqslant L_{3} P^{-\delta_{1}+\alpha^{2}} \cdot \mathrm{P}\left(\Psi_{j}(\alpha)\right)
\end{gathered}
$$

с некоторой постоянной $L_{3}\left(\alpha, \delta_{1}, M_{1}\right)$. Учитьвая условие (16), получим неравенство (24) с параметром $q_{1}=\delta_{1}-\alpha^{2}$.

Чтобы доказать (25), напомним, что по условию леммы

$$
\left|\sigma^{c}\right| \equiv \operatorname{card}\{(j, k): j \neq k,(j, k) \notin \sigma\} \leqslant M_{3} m^{2} P^{-\delta_{3}} .
$$

Поэтому при любом $\alpha^{2}<\delta_{1}$ получим (см. (27))

$$
\sum_{(j, k) \in \sigma^{c}} \mathrm{P}\left(U_{j} \cap U_{k}\right) \leqslant M_{3} m^{2} P^{-\delta_{3}} \max _{1 \leqslant j \leqslant m} \mathrm{P}\left(U_{j}\right) \leqslant L_{4} m^{2} P^{-\delta_{3}} \frac{P^{-\alpha^{2} / 2}}{\sqrt{\log P}}
$$

с некоторой константой $L_{4}\left(\alpha, \delta_{1}, \delta_{3}, M_{1}, M_{3}\right)$. Учитьвая (26), (27), при $\alpha^{2}<\min \left\{\delta_{1}, \delta_{3}\right\}$ имеем

$$
\sum_{(j, k) \in \sigma^{c}} \mathrm{P}\left(U_{j}(\alpha) \cap U_{k}(\alpha)\right) \leqslant L_{5} P^{-\delta_{3}+\alpha^{2}}\left(\sum_{j=1}^{m} \mathrm{P}\left(\Psi_{j}(\alpha)\right)\right)^{2}
$$

и, следовательно, при доказательстве (25) мы можем не учитьвать сумму по $\sigma^{c}$ в левой части неравенства.

Заметим, что оценка (27) при $\alpha^{2}<\delta_{1}$ влечет следующее неравенство:

$$
\sum_{j=1}^{m} \mathrm{P}\left(U_{j}(\alpha)\right) \leqslant L_{5} P^{-1+\alpha^{2}}\left(\sum_{j=1}^{m} \mathrm{P}\left(\Psi_{j}(\alpha)\right)\right)^{2}
$$

с некоторой константой $L_{5}\left(\alpha, \delta_{1}, M_{1}\right)$. Следовательно, при доказательстве $(25)$ суммирование по парам $\{(j, k): j=k\}$ в левой части также можно не учитывать.

Чтобы установить (25), нам осталось заметить, что из условия (17) следует

$$
\sum_{(j, k) \in \sigma} \mathrm{P}\left(U_{j}(\alpha) \cap U_{k}(\alpha)\right) \leqslant \sum_{(j, k) \in \sigma} \mathrm{P}\left(\Psi_{j}(\alpha) \cap \Psi_{k}(\alpha)\right)+M_{2} m^{2} P^{-\delta_{2}} .
$$

При $\alpha^{2}<\min \left\{\delta_{1}, \delta_{2}\right\}$, с помошью (26) оценим погрешность

$$
M_{2} m^{2} P^{-\delta_{2}} \leqslant L_{6} P^{-5\left(\delta_{2}-\alpha^{2}\right) / 6}\left(\sum_{j=1}^{m} \mathrm{P}\left(\Psi_{j}(\alpha)\right)\right)^{2}
$$

с некоторой константой $L_{6}\left(\alpha, \delta_{1}, \delta_{2}, M_{1}, M_{2}\right)$. Таким образом, неравенство (25) доказано с константами $L_{2}=L_{4}+L_{5}+L_{6}, q_{2}=\min \left\{5\left(\delta_{2}-\alpha^{2}\right) / 6, \delta_{3}-\alpha^{2}\right\}$.

Неравенства (24), (25) вместе с неравенством (15) доказьвают (18) с параметром $q^{\prime}=\min \left\{q_{0}, q_{1}, q_{2}\right\}$, где $q_{0}=\min \left(\left(\delta-2 \alpha^{2}\right) / 3,3 \alpha^{2} / 2\right)$ - степенной параметр в неравенстве (15). Лемма 4 доказана. 
ЗАмЕчАниЕ 3. Несложная модификация доказательства теоремы А с применением лемм 2-4 позволяет получить оценки $(5),(6)$ для произвольного значения степенного параметра $q<3(1-2 p) / 11$. Более того, если мы примем во внимание замечание 2 и немного улучшим оценки в леммах 3 и 4 , то можно доказать оценки $(5),(6)$ при любом значении параметра $q<(1-2 p) / 2$, однако при этом константы $C_{1}, C_{2}, C_{3}$, будут зависеть от $q$. В [16] приведено подробное доказательство для случая $q=(1-2 p) / 4$.

2.4. Центральная предельная теорема. Нам потребуется применить вариант двумерной центральной предельной теоремы с оценкой остаточного члена. Мы используем в одно- и двумерном случаях следующий результат Ротаря [22] (см. также следствие 17.2 в [23]).

ПРЕДЛОЖЕНИЕ 1. Для набора независимых случайных векторов $\left\{X_{i}\right\}_{i=1}^{N}$ в $\mathbb{R}^{d}$ таких, что $\mathrm{E}_{i}=0,1 \leqslant i \leqslant N$, имеет место следующее неравенство:

$$
\sup _{A \in \mathscr{C}}\left|P_{N}(A)-\Phi_{0, V}(A)\right| \leqslant K_{1}(d) N^{-1 / 2} m_{3} \lambda^{-3 / 2}
$$

где $P_{N}(A)$ - вероятность того, что случайный вектор $N^{-1 / 2} \sum_{i=1}^{N} X_{i}$ принадлехит мнохеству $A, \mathscr{C}$ - класс всех выпуклых борелевских подмножсеств $\mathbb{R}^{d}$, $K_{1}(d)<\infty$ - некоторая постоянная,

$$
m_{3}:=\frac{1}{N} \sum_{i=1}^{N} \mathrm{E}\left|X_{i}\right|^{3}
$$

$\lambda$ - наименьшее собственное число матриць $V=N^{-1} \sum_{i=1}^{N} \operatorname{cov}\left(X_{i}\right), \operatorname{cov}\left(X_{i}\right)-$ ковариационная матрича вектора $X_{i}$, наконеи, $\Phi_{0, V}$ обозначает гауссовскую меру на $\mathbb{R}^{d}$ с плотностью

$$
\phi_{0, V}(Y):=(2 \pi)^{-d / 2}(\operatorname{det} V)^{-1 / 2} \exp \left\{-\frac{1}{2}\left(Y, V^{-1} Y\right)\right\}, \quad Y \in \mathbb{R}^{d}
$$

\section{3. Доказательство теоремы 1}

Теорема 1 будет получена как непосредственное следствие более общей теоремы $1^{\prime}$ и неравенства Хинчина.

ТЕОрема 1'. Пусть $\left\{f_{i}\right\}_{i=1}^{n}$ - система интегрируемых функиий на вероятностном пространстве $(X, \mu)$ таких, что

$\left(\mathrm{d}^{\prime}\right)\left\|f_{i}\right\|_{1}=1$ для всех $i=1, \ldots, n,\left\|\sum_{i=1}^{n} \theta_{i} f_{i}\right\|_{1} \leqslant M n^{1 / 2+p_{1}}$ для всех наборов знаков $\theta_{i}= \pm 1 u\left\|\left(\sum_{i=1}^{n}\left|f_{i}\right|^{2}\right)^{1 / 2}\right\|_{1} \leqslant M n^{1 / 2+p_{2}}$ с некоторыми постоянными $M, p_{1}, p_{2} \geqslant 0$, причем $p_{1}+2 p_{2}<1 / 2$ u $p_{2}<1 / 12$.

Пусть $\left\{\xi_{i}\right\}_{i=1}^{n}-$ набор независимых случайных величин на другом вероятностном пространстве $(\Omega, \mathrm{P})$ таких, что $\mathrm{E} \xi_{i}=0, \mathrm{E}\left|\xi_{i}\right|^{2}=1 u \mathrm{E}\left|\xi_{i}\right|^{3} \leqslant M^{3}$. Тогда при любом $m \leqslant n$ для случайного полинома (1) имеют место оценки (7) $u$ (8) $c$ некоторыми константами $q=q\left(p_{1}, p_{2}\right)>0, C_{j}=C_{j}\left(p_{1}, p_{2}, M\right)>0, j=1,2,3$. 
(Эти константы, конечно, не совпадают с константами из формулировки теоремы A.)

Чтобы вывести теорему 1 из теоремы $1^{\prime}$, достаточно заметить, что из свойства (d) и проинтегрированного по $x \in X$ неравенства Хинчина (см., например, [2]) для суммы $\sum r_{i}(\omega) f_{i}(x)$ при фиксированном $x$, где $r_{i}-$ функции Радемахера, следует, что

$$
\left\|\left(\sum_{i=1}^{n}\left|f_{i}\right|^{2}\right)^{1 / 2}\right\|_{1} \leqslant \text { const } \cdot \mathrm{E} \int_{X}\left|\sum_{i=1}^{n} r_{i}(\omega) f_{i}(x)\right| d \mu(x) \leqslant M^{\prime}(M) n^{1 / 2+p} .
$$

Поэтому условие $(\mathrm{d})$ с параметром $p=p_{1}$ влечет условие $\left(\mathrm{d}^{\prime}\right)$ с $p_{2} \leqslant p_{1}$ и, следовательно, если $p_{1}<1 / 12$, то неравенство $p_{1}+2 p_{2}<1 / 2$ вьполняется автоматически.

Теорема $1^{\prime}$, как и теорема A, опирается на центральную предельную теорему (предложение 1), однако требует значительно более тонкой подготовительной работы. Дело, грубо говоря, в том, что предположение равномерной ограниченности базисных функций $\left\{f_{i}\right\}$ в $L_{2+\varepsilon}$ позволяло доказать существование “достаточно большого" множества $E \subset X$, на котором, в частности, вьполнялась оценка

$$
\frac{\sum_{i=1}^{n}\left|f_{i}(x)\right|^{3}}{\left(\sum_{i=1}^{n}\left|f_{i}(x)\right|^{2}\right)^{3 / 2}} \leqslant \text { const } \cdot n^{-\varepsilon}, \quad x \in E
$$

с некоторым $\varepsilon>0$. Это неравенство требуется для оценки погрешности в центральной предельной теореме. Такой прием был использован для доказательства оценок (5), (6) в [3] (а ранее использован в [12], [14] для доказательства аналогичных оценок для равномерной нормы).

Однако в предположениях теоремы $1^{\prime}$ точек $x \in X$, для которых имеет место (28), может вообще не существовать. В качестве соответствующего примера можно привести набор (нормированных в $L_{1}$ ) функций $f_{i}:=r_{i}+n^{q} \chi_{i}$, где $r_{i}$ фуннкции Радемахера, а $\chi_{i}$ - характеристические функции интервалов $((i-1) / n, i / n), 1 / 3<q<7 / 12$. Для таких функций имеет место (d) c $p=\max \{0, q-1 / 2\}<1 / 12$, но неравенство (28) нарушается п.в. на $[0,1]$. Поэтому применить предложение 1, как, впрочем, и другие версии центральной предельной теоремы, к суммам вида $\sum_{1}^{n} \xi_{i} f_{i}(x)$ напрямую нельзя. Тем не менее, условие $\left(\mathrm{d}^{\prime}\right)$ оставляет возможность для “достаточно большого" множества точек $x \in X$ выделить “достаточно большую" подсумму $\sum_{i \in \mathscr{I}_{x}} \xi_{i} f_{i}(x)$, для которой оценка (28) выполнена и, следовательно, применима центральная предельная теорема. При этом множество индексов $\mathscr{I}_{x}$, вообше говоря, зависит от $x$, и поэтому возникают трудности, связанные с переносом полученных оценок для подсумм на случай исходного полинома.

ДоКАЗАТЕЛЬСТВО ТЕОРЕМЫ $1^{\prime}$. Не умаляя общности, мы можем считать, что $2 \leqslant m \leqslant n^{\varepsilon_{1}}-1$ с некоторым заранее фиксированным $\varepsilon_{1}=\varepsilon_{1}\left(p_{1}, p_{2}\right) \in(0,1 / 2)$, значение которого будет задано позже. Более того, так как $\|f\|_{m, \infty}$ зависит только от распределения $f$ (см. (4)) и $L_{1}[0,1]$ содержит равноизмеримую копию вектора $\left(f_{j}\right)_{1}^{n}$, мы можем предположить, что $X=[0,1]$ со стандартной мерой Лебега. Последнее означает, что мы сможем использовать теорему 2.

Шаг 1. Для каждого $x \in X$ определим множество индексов

$$
\mathscr{A}(x) \equiv \mathscr{A}_{x}:=\left\{k: 1 \leqslant k \leqslant n,\left|f_{k}(x)\right| \leqslant n^{-1 / 2-\varepsilon_{1}} \sum_{j=1}^{n}\left|f_{j}(x)\right|\right\} .
$$


Из неравенства Чебьшёва получим, что $\left|\mathscr{A}_{x}^{c}\right| \leqslant n^{1 / 2+\varepsilon_{1}}$ и $\left|\mathscr{A}_{x}\right| \geqslant n-n^{1 / 2+\varepsilon_{1}}$. Для каждого $x \in X$ определим также меньшее множество индексов $\Lambda(x) \equiv \Lambda_{x} \subset \mathscr{A}_{x},\left|\Lambda_{x}\right|=$ $n-\left[n^{1 / 2+2 \varepsilon_{1}}\right]$ так, что в каждой точке $x$ множеством $\Lambda_{x}$ индексированы $n-\left[n^{1 / 2+2 \varepsilon_{1}}\right]$ наименьших значений $\left|f_{k}(x)\right|$. Чтобы формально определить множество $\Lambda_{x}$ (такое, что для всех $k \in\{1, \ldots, n\}$ множество $\{x: k \in \Lambda(x)\} \quad \mu$-измеримо), мы можем воспользоваться следующей индукционной процедурой. Положим

$$
\begin{aligned}
& k_{1}(x):=\min \left\{k \in\{1, \ldots, n\}:\left|f_{k}(x)\right| \geqslant\left|f_{i}(x)\right| \forall i=1, \ldots, n\right\}, \\
& \mathscr{K}_{1}(x):=\{1, \ldots, n\} \backslash\left\{k_{1}(x)\right\} .
\end{aligned}
$$

При уже определенных $k_{l}(x), \mathscr{K}_{l}(x), l=1, \ldots, j-1$, положим

$$
\begin{aligned}
k_{j}(x) & :=\min \left\{k \in \mathscr{K}_{j-1}(x):\left|f_{k}(x)\right| \geqslant\left|f_{i}(x)\right| \forall i \in \mathscr{K}_{j-1}(x)\right\}, \\
\mathscr{K}_{j}(x) & :=\mathscr{K}_{j-1}(x) \backslash\left\{k_{j}(x)\right\} .
\end{aligned}
$$

Несложно показать, что индексы $k_{j}(x), j=1, \ldots, n$, являются измеримьми функциями $x$. Положим $\Lambda_{x}:=\{1, \ldots, n\} \backslash \mathscr{K}_{\left[n^{\left.1 / 2+2 \varepsilon_{1}\right]}\right.}(x)$. Легко видеть, что $\left|\Lambda_{x}\right|=n-$ $\left[n^{1 / 2+2 \varepsilon_{1}}\right], \Lambda_{x} \subset \mathscr{A}_{x}$ и

$$
\int_{X} \sum_{k \in \Lambda_{x}^{c}}\left|f_{k}(x)\right| d \mu(x) \leqslant \int_{X} \sqrt{\left|\Lambda_{x}^{c}\right|} \cdot\left(\sum_{k=1}^{n}\left|f_{k}\right|^{2}\right)^{1 / 2} d \mu(x) \leqslant M n^{3 / 4+p_{2}+\varepsilon_{1}}
$$

и, следовательно,

$$
\int_{X} \sum_{k \in \Lambda_{x}}\left|f_{k}(x)\right| d \mu(x) \geqslant n-M n^{3 / 4+p_{2}+\varepsilon_{1}} .
$$

Нетривиальность этой оценки обеспечивается таким выбором $\varepsilon_{1}$, что $3 / 4+p_{2}+\varepsilon_{1}<1$.

Шаг 2. Положим

$$
X^{\prime}:=\left\{x \in X: \sum_{k \in \Lambda_{x}}\left|f_{k}(x)\right| \geqslant \frac{1}{3} \sum_{k=1}^{n}\left|f_{k}(x)\right|\right\}
$$

и заметим, что

$$
\int_{X \backslash X^{\prime}} \sum_{k \in \Lambda_{x}}\left|f_{k}\right| \leqslant \frac{1}{3} \int_{X} \sum_{k=1}^{n}\left|f_{k}\right|=\frac{n}{3} .
$$

Следовательно, для достаточно больших ${ }^{4} n \geqslant n_{0}\left(\varepsilon_{1}+p_{2}, M\right)$ имеем

$$
\int_{X^{\prime}} \sum_{\Lambda_{x}}\left|f_{k}\right| \geqslant n-M n^{3 / 4+p_{2}+\varepsilon_{1}}-\frac{n}{3} \geqslant \frac{n}{2} .
$$

С помощью неравенства Гёльдера для функции

$$
F_{2}(x):=\left(\sum_{k \in \Lambda_{x}}\left|f_{k}(x)\right|^{2}\right)^{1 / 2}
$$

\footnotetext{
${ }^{4}$ Как обычно, случай малых $n$ можно исключить из рассмотрения, увеличив или уменьшив значения некоторых констант.
} 
получим

$$
\int_{X^{\prime}} F_{2}(x) d \mu(x) \geqslant \int_{X^{\prime}}\left|\Lambda_{x}\right|^{-1 / 2} \cdot \sum_{k \in \Lambda_{x}}\left|f_{k}\right| d \mu(x) \geqslant \frac{\sqrt{n}}{2} .
$$

Заметим, если $x \in X^{\prime}$ и некоторое множество индексов $I_{x} \subset \mathscr{A}_{x}$ обладает свойством $\left|I_{x}\right| \geqslant\left|\Lambda_{x}\right|$, то в силу определений множеств $\Lambda_{x}$ и $\mathscr{A}_{x}$ получим

$$
\begin{aligned}
\sum_{k \in I_{x}}\left|f_{k}(x)\right|^{3} & \leqslant\left(\sum_{k \in I_{x}}\left|f_{k}(x)\right|^{2}\right) \cdot \max _{k \in I_{x}}\left\{\left|f_{k}(x)\right|\right\} \\
& \leqslant\left(\sum_{k \in I_{x}}\left|f_{k}(x)\right|^{2}\right) \cdot n^{-1 / 2-\varepsilon_{1}} \sum_{k=1}^{n}\left|f_{k}(x)\right| \\
& \leqslant n^{-1 / 2-\varepsilon_{1}}\left(\sum_{k \in I_{x}}\left|f_{k}(x)\right|^{2}\right) \cdot 3\left(\sum_{k \in \Lambda_{x}}\left|f_{k}(x)\right|\right) \\
& \leqslant 3 n^{-1 / 2-\varepsilon_{1}}\left|\Lambda_{x}\right|^{1 / 2}\left(\sum_{k \in I_{x}}\left|f_{k}(x)\right|^{2}\right)^{3 / 2} \leqslant 3 n^{-\varepsilon_{1}}\left(\sum_{k \in I_{x}}\left|f_{k}(x)\right|^{2}\right)^{3 / 2} .
\end{aligned}
$$

Значит,

$$
\frac{\sum_{k \in I_{x}}\left|f_{k}(x)\right|^{3}}{\left(\sum_{k \in I_{x}}\left|f_{k}(x)\right|^{2}\right)^{3 / 2}} \leqslant 3 n^{-\varepsilon_{1}}
$$

Это неравенство потребуется в дальнейшем для оценки погрешности остаточного члена в центральной предельной теореме.

Шаг 3. Определим множества

$$
E_{\ell}:=\left\{x \in X^{\prime}: \frac{\sqrt{n}}{4} 2^{\ell-1} \leqslant F_{2}(x)<\frac{\sqrt{n}}{4} 2^{\ell}\right\}, \quad \ell=1,2, \ldots .
$$

Считая $n$ достаточно большим, чтобы вьполнялось неравенство

$$
\frac{\sqrt{n}}{2} \leqslant \int_{X^{\prime}} F_{2} \leqslant M n^{1 / 2+p_{2}}
$$

получим для любого $\varepsilon_{2}>0$

$$
\mu\left\{x \in X^{\prime}: F_{2}(x) \geqslant \frac{\sqrt{n}}{4} 2^{n^{\varepsilon_{2}}}\right\} \leqslant \frac{4}{\sqrt{n}} 2^{-n^{\varepsilon_{2}}} \int_{X^{\prime}} F_{2} d \mu<4 M n^{p_{2}} 2^{-n^{\varepsilon_{2}}} .
$$

Заметим, что

$$
\sum_{\ell=1}^{\infty} \int_{E_{\ell}} F_{2} d \mu=\int_{\left\{F_{2} \geqslant \sqrt{n} / 4\right\} \cap X^{\prime}} F_{2} d \mu \geqslant \frac{\sqrt{n}}{4}
$$

и, следовательно, заведомо имеет место хотя бы один из следующих двух случаев:

(i) $\sum_{1 \leqslant \ell<n^{\varepsilon_{2}}} \int_{E_{\ell}} F_{2} \geqslant \frac{\sqrt{n}}{8}$;

(ii) $\sum_{\ell \geqslant n^{\varepsilon_{2}}} \int_{E_{\ell}} F_{2}>\frac{\sqrt{n}}{8}$. 
Предположим сначала, что имеет место (i). Определим множество индексов

$$
\mathscr{L}:=\left\{1 \leqslant \ell<n^{\varepsilon_{2}}: \int_{E_{\ell}} F_{2} \geqslant \frac{n^{1 / 2-\varepsilon_{2}}}{16}\right\}
$$

и заметим, что $\sum_{\ell \in \mathscr{L}} \int_{E_{\ell}} F_{2} \geqslant \sqrt{n} / 16$. Обозначим

$$
\mu_{\ell}:=\mu E_{\ell}, \quad \rho_{\ell}:=\frac{\sqrt{n}}{4} 2^{\ell-1} .
$$

Легко видеть, что в случае (i) мы имеем

$$
\sum_{\ell \in \mathscr{L}} \mu_{\ell} \rho_{\ell} \geqslant \frac{\sqrt{n}}{32}, \quad \mu_{\ell} \rho_{\ell} \geqslant \frac{n^{1 / 2-\varepsilon_{2}}}{32} \quad \text { для всех } \ell \in \mathscr{L} \text {. }
$$

Далее в шагах 5-11 мы будем иметь дело только со случаем (i). Случай (ii) проще, и мы рассмотрим его на заключительном шаге 12 .

Шаг 4. Пусть $\mathscr{J}_{x}$ - некоторое (зависящее от $x \in X$ ) множество индексов (вложенное в $\{1, \ldots, n\})$ такое, что $\left|\mathscr{J}_{x}\right| \geqslant n-n^{1 / 2+2 \varepsilon}, \varepsilon<1 / 4$, и для любого $k_{0}$ множество $\left\{x \in X: k_{0} \in \mathscr{J}_{x}\right\}$ измеримо относительно меры $\mu$. Тогда для любого набора знаков $\theta_{k}= \pm 1, k=1, \ldots, n$, имеем

$$
\begin{aligned}
\int_{X}\left|\sum_{k \in \mathscr{J}_{x}} \theta_{k} f_{k}(x)\right| d \mu(x) & \leqslant \int_{X}\left|\sum_{k=1}^{n} \theta_{k} f_{k}(x)\right| d \mu(x)+\int_{X}\left|\sum_{k \in \mathscr{J}_{x}^{c}} \theta_{k} f_{k}(x)\right| d \mu(x) \\
& \leqslant M n^{1 / 2+p_{1}}+\int_{X} \sqrt{\left|\mathscr{J}_{x}^{c}\right|} \cdot\left(\sum_{k=1}^{n}\left|f_{k}(x)\right|^{2}\right)^{1 / 2} d \mu(x) \\
& \leqslant M n^{1 / 2+p_{1}}+M n^{3 / 4+p_{2}+\varepsilon} \leqslant 2 M n^{1 / 2+\max \left\{p_{1}, p_{2}+1 / 4+\varepsilon\right\}} .
\end{aligned}
$$

Следовательно, по лемме 1 для всех коэффициентов $\left\{a_{k}\right\}_{1}^{n}$ имеем

$$
\int_{X}\left|\sum_{k \in \mathscr{J}_{x}} a_{k} f_{k}\right| d \mu(x) \leqslant C n^{1 / 4+(1 / 2) \max \left\{p_{1}, p_{2}+1 / 4+\varepsilon\right\}}\left(\sum_{k=1}^{n}\left|a_{k}\right|^{2}\right)^{1 / 2}
$$

с некоторой константой $C(M)>0$.

Шаг 5. Выберем константы $\varepsilon_{1}, \varepsilon_{2}, \varepsilon_{3}>0$ так, чтобы выполнялись следующие неравенства:

$$
2 \varepsilon_{2}+\varepsilon_{3} \leqslant \frac{1}{4}\left(1-2 p_{1}-4 p_{2}\right), \quad \frac{\varepsilon_{1}}{2}+2 \varepsilon_{2}+\varepsilon_{3} \leqslant \frac{3}{2}\left(\frac{1}{12}-p_{2}\right) .
$$

Смысл этих неравенств вскоре прояснится. Мы можем для определенности положить

$$
2 \varepsilon_{2}=\frac{\varepsilon_{1}}{2}=\varepsilon_{3}:=\min \left\{\frac{1}{2}\left(\frac{1}{12}-p_{2}\right), \frac{1}{8}\left(1-2 p_{1}-4 p_{2}\right)\right\} .
$$

Положительность $\varepsilon_{1}, \varepsilon_{2}, \varepsilon_{3}$ обеспечивается ограничениями, наложенньми на $p_{1}$ и $p_{2}$. 
Рассмотрим некоторое $E_{\ell}, \ell \in \mathscr{L}$. Для $\bar{x}=\left(x_{i}\right)_{1}^{m} \in\left(E_{\ell}\right)^{m}$ положим

$$
\begin{aligned}
& \mathscr{I}\left(x_{1}, \ldots, x_{m}\right):=\bigcap_{j=1}^{m} \mathscr{A}_{x_{j}}, \\
& \varphi\left(x_{1}, \ldots, x_{m}\right):=\frac{1}{m^{2}} \sum_{\substack{j, k=1 \\
j \neq k}}^{m}\left|\sum_{i \in \mathscr{I}(\bar{x})} f_{i}\left(x_{j}\right) f_{i}\left(x_{k}\right)\right| .
\end{aligned}
$$

Заметим, что $|\mathscr{I}(\bar{x})| \geqslant n-m n^{1 / 2+\varepsilon_{1}} \geqslant n-n^{1 / 2+2 \varepsilon_{1}}$. Пользуясь инвариантностью множества $\mathscr{I}\left(x_{1}, \ldots, x_{m}\right)$ и функции $\varphi\left(x_{1}, \ldots, x_{m}\right)$ относительно перестановок координат $\left(x_{1}, \ldots, x_{m}\right)$, получим

$$
\begin{aligned}
& \frac{1}{\mu_{\ell}^{m}} \int_{\left(E_{\ell}\right)^{m}} \varphi(\bar{x}) d \mu^{m}(\bar{x})=\frac{m(m-1)}{m^{2} \mu_{\ell}^{m}} \int_{\left(E_{\ell}\right)^{m}}\left|\sum_{k \in \mathscr{I}(\bar{x})} f_{k}\left(x_{1}\right) f_{k}\left(x_{2}\right)\right| d \mu^{m}(\bar{x}) \\
& \quad \leqslant \frac{1}{\mu_{\ell}^{m}} \int_{\left(E_{\ell}\right)^{m-2}}\left\{\int_{X} \int_{X}\left|\sum_{k \in \mathscr{I}(\bar{x})} f_{k}\left(x_{1}\right) f_{k}\left(x_{2}\right)\right| d \mu\left(x_{1}\right) d \mu\left(x_{2}\right)\right\} d \mu^{m-2}\left(x_{3}, \ldots, x_{m}\right) .
\end{aligned}
$$

Применяя результаты шага 4 к множеству $\mathscr{I}(\bar{x})$ с $\varepsilon=\varepsilon_{1}$, получим

$$
\begin{aligned}
& \int_{X} \int_{X}\left|\sum_{k \in \mathscr{I}(\bar{x})} f_{k}\left(x_{1}\right) f_{k}\left(x_{2}\right)\right| d \mu\left(x_{1}\right) d \mu\left(x_{2}\right) \\
& \leqslant C n^{1 / 4+\max \left\{p_{1}, p_{2}+1 / 4+\varepsilon_{1}\right\} / 2} \cdot \int_{X}\left(\sum_{k=1}^{n}\left|f_{k}\left(x_{1}\right)\right|^{2}\right)^{1 / 2} d \mu\left(x_{1}\right),
\end{aligned}
$$

из чего окончательно получим

$$
\frac{1}{\mu_{\ell}^{m}} \int_{\left(E_{\ell}\right)^{m}} \varphi(\bar{x}) d \mu\left(x_{1}\right) \ldots d \mu\left(x_{m}\right) \leqslant \frac{C M}{\mu_{\ell}^{2}} n^{3 / 4+p_{2}+\max \left\{p_{1}, p_{2}+1 / 4+\varepsilon_{1}\right\} / 2} .
$$

Мы хотим доказать существование множества $G_{\ell} \subset\left(E_{\ell}\right)^{m}$ такого, что $\mu^{m} G_{\ell} \geqslant \mu_{\ell}^{m} / 2$ и для любого $\bar{x}=\left(x_{i}\right)_{1}^{m} \in G_{\ell}$ имеет место

$$
\varphi(\bar{x}) \leqslant C_{0} \rho_{\ell}^{2} n^{-\varepsilon_{3}}
$$

с некоторыми константами $\varepsilon_{3}, C_{0}\left(p_{1}, p_{2}, M\right)>0$. Для этого заметим, что при $\ell \in \mathscr{L}$ мы имеем $\mu_{\ell} \rho_{\ell} \geqslant 2^{-5} n^{1 / 2-\varepsilon_{2}}$, что вместе с (32) влечет

$$
\begin{aligned}
\frac{1}{\mu_{\ell}^{m}} \int_{\left(E_{\ell}\right)^{m}} \varphi(\bar{x}) d \mu^{m}(\bar{x}) & \leqslant C M 2^{10} \rho_{\ell}^{2} n^{2 \varepsilon_{2}-1} n^{3 / 4+p_{2}+\max \left\{p_{1}, p_{2}+1 / 4+\varepsilon_{1}\right\} / 2} \\
& \leqslant C M 2^{10} \rho_{\ell}^{2} n^{-\varepsilon_{3}}
\end{aligned}
$$

где константы $\varepsilon_{1}, \varepsilon_{2}, \varepsilon_{3}$ выбраны положительньми, удовлетворяющими неравенствам (31). Таким образом, с помошью неравенства Чебышёва для множества

$$
G_{\ell}:=\left\{\bar{x} \in E_{\ell}: \varphi(\bar{x}) \leqslant C M 2^{11} \rho_{\ell}^{2} n^{-\varepsilon_{3}}\right\}, \quad \ell \in \mathscr{L},
$$

имеем $\mu^{m} G_{\ell} \geqslant \mu_{\ell}^{m} / 2$. 
Шаг 6. Для $\ell \in \mathscr{L}_{\text {и }}\left(x_{j}\right)_{1}^{m} \in G_{\ell}$ определим случайный вектор $\eta=\left(\eta_{j}\right)_{1}^{m}$ тождеством

$$
\eta_{j}(\omega):=|\mathscr{I}(\bar{x})|^{-1 / 2} \sum_{i \in \mathscr{I}\left(x_{1}, \ldots, x_{m}\right)} \xi_{i}(\omega) f_{i}\left(x_{j}\right)
$$

Мы хотим показать, что

$$
\sup _{v \in \mathbb{R}^{m}} \mathrm{P}\left\{\max _{1 \leqslant j \leqslant m}\left|\eta_{j}+v_{j}\right| \leqslant \alpha \rho_{\ell} \sqrt{\frac{\log m}{|\mathscr{I}(\bar{x})|}}\right\} \leqslant \frac{K_{0}}{m^{q}}
$$

с некоторыми положительньми постоянньми $\alpha, K_{0}$ и $q$, зависящими только от $p_{1}, p_{2}, M$. Нетрудно проверить, что неравенство (33) вьполнено, если

$$
\min _{\theta_{j}= \pm 1} \mathrm{P}\left(\bigcup_{j=1}^{m}\left\{\theta_{j} \eta_{j} \geqslant \alpha \rho_{\ell}\left(\frac{\log m}{|\mathscr{I}(\bar{x})|}\right)^{1 / 2}\right\}\right) \geqslant 1-\frac{K_{0}}{m^{q}}
$$

(достаточно взять $\theta_{j}=-\operatorname{sign}\left(v_{j}\right)$ ). Последнее неравенство мы установим, показав, что

$$
\begin{gathered}
\min _{\theta_{j}= \pm 1} \mathrm{P}\left(\bigcup_{j=1}^{m}\left\{\theta_{j} \eta_{j} \geqslant \alpha \sqrt{d_{j, \ell} \log m}\right\}\right) \geqslant 1-\frac{K_{0}}{m^{q}}, \\
\text { где } d_{j, \ell}:=\mathrm{D}\left(\eta_{j}\right)=\frac{1}{|\mathscr{I}(\bar{x})|} \sum_{i \in \mathscr{I}(\bar{x})}\left|f_{i}\left(x_{j}\right)\right|^{2} \geqslant \frac{1}{|\mathscr{I}(\bar{x})|} \sum_{i \in \Lambda\left(x_{j}\right)}\left|f_{i}\left(x_{j}\right)\right|^{2} \geqslant \frac{\rho_{\ell}^{2}}{|\mathscr{I}(\bar{x})|} .
\end{gathered}
$$

Оценивая $d_{j, \ell}$, мы воспользовались определением $\Lambda_{x}$ и тем фактом, что

$$
|\mathscr{I}(\bar{x})| \geqslant n-m \cdot n^{1 / 2+2 \varepsilon_{1}} \geqslant\left|\Lambda_{x}\right| .
$$

Для фиксированного набора знаков $\left\{\theta_{j}\right\}_{1}^{m}, \theta_{j}= \pm 1$, обозначим

$$
U_{j}=U_{j}(\alpha):=\left\{\omega \in \Omega: \theta_{j} \eta_{j} \geqslant \alpha \sqrt{d_{j, \ell} \log m}\right\}, \quad v_{j, k}:=\frac{\theta_{j} \theta_{k}}{|\mathscr{I}(\bar{x})|} \sum_{i \in \mathscr{I}(\bar{x})} f_{i}\left(x_{j}\right) f_{i}\left(x_{k}\right)
$$

и заметим, что по определению множества $G_{\ell}$

$$
\frac{1}{m^{2}} \sum_{\substack{j, k=1 \\ j \neq k}}^{m}\left|v_{j, k}\right| \leqslant C_{0} \rho_{\ell}^{2} n^{-\varepsilon_{3}}|\mathscr{I}(\bar{x})|^{-1} .
$$

Далее, с помошью центральной предельной теоремы мы собираемся показать, что случайные величины $\eta_{j}$ "почти" гауссовские и, более того, они “почти" попарно гауссовские. Затем, мы применим лемму 4 (с параметрами $R=n, P=m$ ) и установим для событий $U_{j}$ неравенство

$$
\sum_{j, k=1}^{m} \mathrm{P}\left(U_{j}(\alpha) \cap U_{k}(\alpha)\right) \leqslant\left(1+L_{0} m^{-q}\right)\left(\sum_{j=1}^{m} \mathrm{P}\left(U_{j}(\alpha)\right)\right)^{2}
$$

с некоторыми константами $L_{0}\left(p_{1}, p_{2}, M\right), q\left(p_{1}, p_{2}\right)>0$, из которого с помощью леммы 2 выводится неравенство (34). Таким образом, чтобы доказать (33) нам достаточно доказать (36), доказательству (36) посвящены шаги 7 и 8. 
Шаг 7. Пусть $\left(h_{j}\right)_{1}^{m}$ обозначает гауссовский вектор с нулевьм средним и ковариациями $\mathrm{E} h_{j} h_{k}=v_{j, k}$ и $\Psi_{j}=\Psi_{j}(\alpha):=\left\{\omega: h_{j}>\alpha \sqrt{d_{j, \ell} \log m}\right\}$. Заметим, что

$$
\mathrm{P}\left(\Psi_{j}\right)=\left(2 \pi d_{j, \ell}\right)^{-1 / 2} \int_{\alpha \sqrt{d_{j, \ell} \log m}}^{\infty} \exp \left(-\frac{y^{2}}{2 d_{j, \ell}}\right) d y .
$$

Мы собираемся применить к случайньм векторам $\left(\eta_{j}\right)_{1}^{m}$ и $\left(h_{j}\right)_{1}^{m}$ лемму 4 . Чтобы показать вьполнение условий (16), (17), мы воспользуемся предложением 1.

Зафиксируем некоторую точку $\bar{x}=\left(x_{j}\right)_{1}^{m} \in G_{\ell}$ и набор знаков $\left\{\theta_{j}\right\}_{1}^{m}, \theta_{j}= \pm 1$, и применим предложение 1 к наборам случайных величин $\left\{\theta_{j} f_{i}\left(x_{j}\right) \xi_{i}(\omega)\right\}_{i \in \mathscr{I}(\bar{x})}$ при всех $1 \leqslant j \leqslant m$. Здесь $N=|\mathscr{I}(\bar{x})|$ и

$$
\begin{gathered}
m_{3}=\frac{1}{|\mathscr{I}(\bar{x})|} \sum_{k \in \mathscr{I}(\bar{x})} \mathrm{E}\left|\xi_{k}\right|^{3}\left|f_{k}\left(x_{j}\right)\right|^{3} \leqslant \frac{M^{3}}{|\mathscr{I}(\bar{x})|} \sum_{k \in \mathscr{I}(\bar{x})}\left|f_{k}\left(x_{j}\right)\right|^{3} \\
\lambda=V=\frac{1}{|\mathscr{I}(\bar{x})|} \sum_{k \in \mathscr{I}(\bar{x})} \mathrm{E}\left|\xi_{k}\right|^{2}\left|f_{k}\left(x_{j}\right)\right|^{2}=d_{j, \ell} .
\end{gathered}
$$

Учитьвая неравенство (29), получим

$$
|\mathscr{I}(\bar{x})|^{-1 / 2} m_{3} \lambda^{-3 / 2} \leqslant 3 M^{3} n^{-\varepsilon_{1}}
$$

и, следовательно, предложение 1 влечет для случайной величины $\theta_{j} \eta_{j}$ следующую оценкy:

$$
\begin{aligned}
\left|\mathrm{P}\left(U_{j}(\alpha)\right)-\mathrm{P}\left(\Psi_{j}(\alpha)\right)\right| & \equiv\left|\mathrm{P}\left(U_{j}(\alpha)\right)-\left(\frac{1}{2 \pi d_{j, \ell}}\right)^{1 / 2} \int_{\alpha \sqrt{d_{j, \ell} \log m}}^{\infty} \exp \left\{\frac{-y^{2}}{2 d_{j, \ell}}\right\} d y\right| \\
& \leqslant 3 K_{1}(1) M^{3} n^{-\varepsilon_{1}}
\end{aligned}
$$

Шаг 8. Как и в доказательстве леммы 3 , положим

$$
\sigma_{1}:=\left\{(j, k): 1 \leqslant j \neq k \leqslant m,\left|v_{j, k}\right|<\frac{\rho_{\ell}^{2}}{8|\mathscr{I}(\bar{x})|}\right\} .
$$

Из неравенства Чебьшёва следует, что (см. (35))

$$
\left|\sigma_{1}^{c}\right| \leqslant \frac{8|\mathscr{I}(\bar{x})|}{\rho_{\ell}^{2}} \sum_{\substack{j, k=1 \\ j \neq k}}^{m}\left|v_{j, k}\right| \leqslant 8 C_{0} m^{2} n^{-\varepsilon_{3}} \leqslant 8 C_{0} m^{2-\varepsilon_{3} / \varepsilon_{1}} .
$$

(Мы воспользовались предположением, что $m \leqslant n^{\varepsilon_{1}}$.)

Чтобы применить лемму 4 и тем самьм доказать (36), нам осталось показать, что при всех $(j, k) \in \sigma_{1}$ вьполнена оценка типа (17).

Для фиксированной пары $s=(j, k) \in \sigma_{1}$ рассмотрим набор случайных векторов в $\mathbb{R}^{2}$

$$
\left\{\left(\frac{\theta_{j} \xi_{i}(\omega) f_{i}\left(x_{j}\right)}{\sqrt{d_{j, \ell}}}, \frac{\theta_{k} \xi_{i}(\omega) f_{i}\left(x_{k}\right)}{\sqrt{d_{k, \ell}}}\right)\right\}_{i \in \mathscr{I}\left(x_{1}, \ldots, x_{m}\right)} .
$$


Применим к этому набору предложение 1. Здесь

$$
\begin{aligned}
m_{3}^{s} & =\frac{1}{|\mathscr{I}(\bar{x})|} \sum_{i \in \mathscr{I}(\bar{x})} \mathrm{E}\left|\xi_{i}\right|^{3}\left(\frac{\left|f_{i}\left(x_{j}\right)\right|^{2}}{d_{j, \ell}}+\frac{\left|f_{i}\left(x_{k}\right)\right|^{2}}{d_{k, \ell}}\right)^{3 / 2}, \\
V^{s} & =\frac{1}{|\mathscr{I}(\bar{x})|}\left(\begin{array}{cc}
\sum_{i \in \mathscr{I}(\bar{x})} \frac{\left|f_{i}\left(x_{j}\right)\right|^{2}}{d_{j, \ell}} & \frac{\theta_{j} \theta_{k}}{\sqrt{d_{j, \ell} d_{k, \ell}}} \sum_{i \in \mathscr{I}(\bar{x})} f_{i}\left(x_{j}\right) f_{i}\left(x_{k}\right) \\
\frac{\theta_{j} \theta_{k}}{\sqrt{d_{j, \ell} d_{k, \ell}}} \sum_{i \in \mathscr{I}(\bar{x})} f_{i}\left(x_{j}\right) f_{i}\left(x_{k}\right) & \sum_{i \in \mathscr{I}(\bar{x})} \frac{\left|f_{i}\left(x_{k}\right)\right|^{2}}{d_{k, \ell}}
\end{array}\right)
\end{aligned}
$$

Следовательно,

$$
V^{s}=\left(\begin{array}{cc}
1 & \frac{v_{s}}{\sqrt{d_{j, \ell} d_{k, \ell}}} \\
\frac{v_{s}}{\sqrt{d_{j, \ell} d_{k, \ell}}} & 1
\end{array}\right)
$$

Пользуясь тем, что $s \in \sigma_{1}$, оценим определитель

$$
\operatorname{det} V^{s}=1-\frac{v_{s}^{2}}{d_{j, \ell} d_{k, \ell}} \geqslant 1-\frac{1}{64} \geqslant \frac{1}{2} .
$$

Поскольку матрица $V^{s}$ положительно определена, оба ее собственных числа $\lambda_{2} \geqslant \lambda_{1}$ положительны. Следовательно, принимая во внимание, что $\lambda_{1}+\lambda_{2}=\operatorname{trace} V^{s}=2$, получим

$$
\frac{1}{2} \leqslant \operatorname{det} V^{s}=\lambda_{2} \lambda_{1} \leqslant 2 \lambda_{1},
$$

следовательно, $\lambda_{1}>1 / 4$. Учитьвая $(25)$ и определение $d_{j, \ell}, \mathscr{I}(\bar{x})$, оценим

$$
m_{3}^{s} \leqslant 8 M^{3}|\mathscr{I}(\bar{x})|^{1 / 2} \cdot 3 n^{-\varepsilon_{1}} .
$$

Таким образом, с помощью предложения 1 имеем

$$
\begin{aligned}
& \left|\mathrm{P}\left(U_{j} \cap U_{k}\right)-\frac{1}{2 \pi \sqrt{\operatorname{det} V^{s}}} \int_{\alpha \sqrt{d_{j, \ell} \log m}}^{\infty} \int_{\alpha \sqrt{d_{k, \ell} \log m}}^{\infty} e^{-\left(Y,\left(V^{s}\right)^{-1} Y\right) / 2} d y_{1} d y_{2}\right| \\
& \equiv\left|\mathrm{P}\left(U_{j} \cap U_{k}\right)-\mathrm{P}\left(\Psi_{j} \cap \Psi_{k}\right)\right| \leqslant \frac{K_{1}(2) m_{3}^{s} \lambda_{1}^{-3 / 2}}{|\mathscr{I}(\bar{x})|^{1 / 2}}<\frac{200 M^{3} K_{1}(2)}{n^{\varepsilon_{1}}} .
\end{aligned}
$$

Следовательно, учитьвая оценки (37)-(39), мы можем воспользоваться леммой 4 (с параметрами $R=n, P=m$ и $\left.\delta_{1}=\delta_{2}=\varepsilon_{1}, \delta=\delta_{3}=\varepsilon_{3} / \varepsilon_{1}\right)$ и получить оценку (36) и, следовательно, установить (33) с некоторыми ${ }^{5}$ фиксированньми $\alpha>0, q\left(p_{1}, p_{2}\right)>0$.

Шаг 9. Целью этого шага будет доказательство неравенства

$$
\mathrm{P}\left(\bigcup_{j=1}^{m}\left\{\omega:\left|\sum_{i=1}^{n} \xi_{i}(\omega) f_{i}\left(x_{j}\right)\right| \leqslant \alpha \rho_{\ell} \sqrt{\log m}\right\}\right) \leqslant K_{0} m^{-q}
$$

для $\left(x_{j}\right)_{1}^{m} \in G_{\ell}, \ell \in \mathscr{L}$. Это неравенство легко вытекает из (33) и следующей леммы.

\footnotetext{
${ }^{5}$ Мы не задаемся здесь вопросом оптимального выбора степенного параметра $q$, заметим только, что при $\alpha^{2}=\left(1 / 12-p_{1}\right) / 4$ лемма 4 гарантирует выполнение неравенств $(36),(33)$ с константой $q=\left(1 / 12-p_{1}\right) / 4$.
} 
ЛЕмма 5. Пусть случайные векторы $\eta=\left(\eta_{j}\right)_{1}^{m} u \eta^{c}=\left(\eta_{j}^{c}\right)_{1}^{m}$ независимы $и$ для некоторого открытого или замкнутого множсества $B \subset \mathbb{R}^{m} u p \in(0,1)$ выполнено неравенство

$$
\sup _{v \in \mathbb{R}^{m}} \mathrm{P}\{\eta+v \in B\} \leqslant p
$$

Tогда $\mathrm{P}\left\{\eta+\eta^{c} \in B\right\} \leqslant p$.

ДокАЗАТЕЛЬСТво ЛЕммы 5 . Пусть $\chi(v, w)$ - характеристическая функция множества $\{(v, w): v+w \in B\} \subset \mathbb{R}^{2 m}$, тогда

$$
\mathrm{P}\left\{\eta+\eta^{c} \in B\right\}=\int_{\mathbb{R}^{m}} \int_{\mathbb{R}^{m}} \chi(v, w) d F_{\eta \mid \eta^{c}}(v \mid w) d F_{\eta^{c}}(w),
$$

где $F_{\eta \mid \eta^{c}}(v \mid w)$ - условное распределение вектора $\eta$ относительно $\eta^{c}$ (определение см. в [24]), $F_{\eta^{c}}(w)$ - распределение вектора $\eta^{c}$. Так как векторы $\eta$ и $\eta^{c}$ независимы, имеем $F_{\eta \mid \eta^{c}}(v \mid w)=F_{\eta}(v)$ и

$$
\mathrm{P}\left\{\eta+\eta^{c} \in B\right\}=\int_{\mathbb{R}^{m}} \int_{\mathbb{R}^{m}} \chi(v, w) d F_{\eta}(v) d F_{\eta^{c}}(w)=\int_{\mathbb{R}^{m}} \mathrm{P}\{\eta \in B-w\} d F_{\eta^{c}}(w) \leqslant p .
$$

Лемма 5 доказана.

Теперь, чтобы вывести $(40)^{6}$ из (33), достаточно заметить, что случайные векторы с координатами

$$
\eta_{j}:=\frac{1}{\sqrt{|\mathscr{I}(\bar{x})|}} \sum_{i \in \mathscr{I}(\bar{x})} \xi_{i} f_{i}\left(x_{j}\right), \quad \eta_{j}^{c}:=\frac{1}{\sqrt{|\mathscr{I}(\bar{x})|}} \sum_{i=1}^{n} \xi_{i} f_{i}\left(x_{j}\right)-\eta_{j}
$$

независимы, и применить к ним лемму 5 с параметром $p=K_{0} m^{-q}$ и $B:=\left\{\left(y_{j}\right)_{1}^{m} \in \mathbb{R}^{m}\right.$ : $\left.\left|y_{j}\right| \leqslant \alpha \rho_{\ell} \sqrt{\log m /|\mathscr{I}(\bar{x})|}\right\}$.

Шаг 10. Для $\ell \in \mathscr{L}$ положим

$$
\Omega_{\ell}:=\left\{\omega \in \Omega: \mu^{m}\left\{\bar{x} \in G_{\ell}: \max _{1 \leqslant j \leqslant m}\left|F_{n}\left(\omega, x_{j}\right)\right| \geqslant \alpha \rho_{\ell} \sqrt{\log m}\right\} \geqslant \frac{\mu^{m} G_{\ell}}{2}\right\} .
$$

Из неравенства (40) очевидно следует, что

$$
\gamma:=\mathrm{P} \times \mu^{m}\left\{(\omega, \bar{x}) \in \Omega \times G_{\ell}: \max _{1 \leqslant j \leqslant m}\left|F_{n}\left(\omega, x_{j}\right)\right| \geqslant \alpha \rho_{\ell} \sqrt{\log m}\right\} \geqslant\left(1-\frac{K_{0}}{m^{q}}\right) \mu^{m} G_{\ell} .
$$

Поэтому, мы можем оценить

$$
\mathrm{P}\left(\Omega_{\ell}\right) \mu^{m} G_{\ell}+\left(1-\mathrm{P}\left(\Omega_{\ell}\right)\right) \frac{\mu^{m} G_{\ell}}{2} \geqslant \gamma \geqslant\left(1-K_{0} m^{-q}\right) \mu^{m} G_{\ell}
$$

и, следовательно, $\mathrm{P}\left(\Omega_{\ell}\right) \geqslant 1-2 K_{0} m^{-q}$.

Нам потребуется следующая

\footnotetext{
${ }^{6}$ Если бы нас интересовала только оценка равномерной нормы случайного полинома (следствие 1), то мы фактически могли бы остановится на этом шаге. В самом деле, неравенство (40) с $m=\left[n^{\varepsilon_{1}}\right]$ доказьвает следствие 1 в случае (i) из шага 3 . Если же имеет место случай (iі), нам достаточно применить предложение 1 к $\left\{f_{i}(x) \xi_{i}\right\}_{i \in \mathscr{A}(x)}$ в единственной точке $x \in E_{\ell}, \ell>n^{\varepsilon_{1}}$, и с помощью леммы 5 получить требуемую оценку с "большим запасом."
} 
Лемма 6. Пусть числа $T_{\ell} \geqslant 0$ таковы, что $\sum_{\ell=1}^{L} T_{\ell}=T$ (L мохет бить бесконечным), а события $\widetilde{\Omega}_{\ell}$ таковы, что $\mathrm{P}\left(\widetilde{\Omega}_{\ell}\right) \geqslant 1-p$. Тогда

$$
\mathrm{P}\left\{\sum_{\ell=1}^{L} T_{\ell} I_{\widetilde{\Omega}_{\ell}}(\omega) \leqslant \frac{T}{2}\right\} \leqslant 2 p
$$

əде $I_{\widetilde{\Omega}_{\ell}}-$ индикаторы событий $\widetilde{\Omega}_{\ell}$.

ДОКАЗАТЕЛЬСТВО ЛЕММЫ 6. Пусть

$$
q=\mathrm{P}\left(\sum_{\ell=1}^{L} T_{\ell} I_{\widetilde{\Omega}_{\ell}} \leqslant \frac{T}{2}\right)
$$

тогда

$$
q \frac{T}{2}+(1-q) T \geqslant \mathrm{E} \sum_{\ell=1}^{L} T_{\ell} I_{\widetilde{\Omega}_{\ell}} \geqslant T(1-p) .
$$

Следовательно, $q \leqslant 2 p$. Лемма 6 доказана.

Применим лемму 6 к числам $T_{\ell}:=\mu_{\ell} \rho_{\ell}$ и событиям $\Omega_{\ell}$ при $\ell \in \mathscr{L}$ с параметром $p=2 K_{0} m^{-q}$. Получим, что существует событие $\Omega_{0}$ и подмножество $\mathscr{L}_{0}(\omega) \subset \mathscr{L}_{\text {такие, }}$ что $\Omega_{0} \subset \bigcap_{\ell \in \mathscr{L}_{0}} \Omega_{\ell}$

$$
\mathrm{P}\left(\Omega_{0}\right) \geqslant 1-4 K_{0} m^{-q}
$$

и п.н. на $\Omega_{0}$ имеет место неравенство (см. шаг 3 )

$$
\sum_{\ell \in \mathscr{L}_{0}(\omega)} \mu_{\ell} \rho_{\ell} \geqslant \frac{1}{2} \sum_{\ell \in \mathscr{L}} \mu_{\ell} \rho_{\ell} \geqslant \frac{\sqrt{n}}{64} .
$$

Шаг 11. Для $\ell \in \mathscr{L}$ положим

$$
\Delta_{\ell}(\omega):=\left\{x \in E_{\ell}:\left|F_{n}(\omega, x)\right| \geqslant \alpha \rho_{\ell} \sqrt{\log m}\right\}
$$

Почти наверное при $\omega \in \Omega_{\ell}$ имеем

$$
\begin{aligned}
\left(\mu E_{\ell}\right)^{m}-\left(\mu E_{\ell}-\mu \Delta_{\ell}\right)^{m} & =\mu^{m}\left\{\bar{x} \in E_{\ell}^{m}: \max _{1 \leqslant j \leqslant m}\left|F_{n}\left(\omega, x_{j}\right)\right| \geqslant \alpha \rho_{\ell} \sqrt{\log m}\right\} \\
& \geqslant \frac{\mu^{m} G_{\ell}}{2} \geqslant \frac{\left(\mu E_{\ell}\right)^{m}}{4} \equiv \frac{\mu_{\ell}^{m}}{4} .
\end{aligned}
$$

Следовательно, $1 \geqslant 1 / 4+\left(1-\mu \Delta_{\ell} / \mu_{\ell}\right)^{m}$ и

$$
\frac{\mu \Delta_{\ell}}{\mu_{\ell}} \geqslant 1-\left(\frac{3}{4}\right)^{1 / m} \geqslant \frac{1}{4 m} .
$$

Сушествуют подмножества $\Delta_{\ell}^{\prime}(\omega) \subset \Delta_{\ell}(\omega)$ такие, что $\mu \Delta_{\ell}^{\prime}=\mu_{\ell} /(4 m)$. Положим

$$
E^{\prime}=\bigcup_{\ell \in \mathscr{L}_{0}} \Delta_{\ell}^{\prime}(\omega), \quad \mu E^{\prime}=\frac{1}{4 m} \sum_{\ell \in \mathscr{L}_{0}} \mu_{\ell} .
$$


Теперь для почти всех $\omega \in \Omega_{0}\left(\Omega_{0}\right.$ и $\mathscr{L}_{0}$ определены в предыдушем шаге) с помошю теоремы 2 (см. (12)) мы можем оценить интегрально-равномерную норму случайного полинома $F_{n}(\omega, x)$ вида $(1)$ с помошью "родственной" ей нормы $\|\cdot\|_{m}^{*}($ см. $(10))$ следуюшим образом:

$$
\begin{aligned}
\left\|F_{n}(\omega, \cdot)\right\|_{m}^{*} & \geqslant \frac{1}{\mu E^{\prime}(\omega)}\left(\int_{E^{\prime}(\omega)}\left|F_{n}(\omega, x)\right| d \mu(x)\right)\left(1-\left(1-\mu E^{\prime}(\omega)\right)^{m}\right) \\
& \geqslant \frac{1}{\mu E^{\prime}(\omega)}\left(\sum_{\ell \in \mathscr{L}_{0}(\omega)} \frac{\mu_{\ell}}{4 m} \alpha \rho_{\ell} \sqrt{\log m}\right)\left(1-\left(1-\mu E^{\prime}(\omega)\right)^{m}\right) \\
& =\alpha \sqrt{\log m}\left(\sum_{\ell \in \mathscr{L}_{0}(\omega)} \mu_{\ell} \rho_{\ell}\right) \cdot t_{0}^{-1}\left(1-\left(1-\frac{t_{0}}{4 m}\right)^{m}\right),
\end{aligned}
$$

где $t_{0}=\sum_{\ell \in \mathscr{L}_{0}} \mu_{\ell}$. Принимая во внимание $(41)$ и то, что $1-(1-t /(4 m))^{m} \geqslant t / 8$ при $t \in(0,1)$ и $m \geqslant 1$, с помошью теоремы 2 получим

$$
\left\|F_{n}(\omega, \cdot)\right\|_{m, \infty} \geqslant\left\|F_{n}(\omega, \cdot)\right\|_{m}^{*} \geqslant 8^{-3} \alpha \sqrt{n \log m} \quad \text { п.н. на } \Omega_{0},
$$

что с учетом оценки $\mathrm{P}\left(\Omega_{0}\right) \geqslant 1-4 K_{0} m^{-q}$ доказывает $(7)$ в том случае, когда имеет место случай (i) (см. шаг 3$)$.

Шаг 12. Чтобы завершить доказательство теоремы, осталось рассмотреть случай (ii) из шага 3. Заметим, что так как неравенство (25) вьполнено п.в. на $X^{\prime}$ (при $I_{x}=\mathscr{A}_{x}$ ), мы можем применить предложение 1 (в одномерном случае) к сумме случайных величин $\eta_{x}:=\left|\mathscr{A}_{x}\right|^{-1 / 2} \sum_{i \in \mathscr{A}_{x}} \xi_{i} f_{i}(x)$. Получим

$$
\sup _{v \in \mathbb{R}} \mathrm{P}\left\{\left|\eta_{x}+v\right| \leqslant \gamma \sqrt{d(x)}\right\}=(2 \pi)^{-1 / 2} \int_{-\gamma}^{\gamma} e^{-y^{2} / 2} d y+O\left(n^{-\varepsilon_{1}}\right),
$$

где $d(x):=\left|\mathscr{A}_{x}\right|^{-1} \sum_{i \in \mathscr{A}_{x}}\left|f_{i}(x)\right|^{2}$. Положим $\gamma=m^{-\varepsilon_{1}}$, тогда для п.в. $x \in E_{\ell}, \ell \geqslant 1$, имеем

$$
\sup _{v \in \mathbb{R}} \mathrm{P}\left\{\left|\eta_{x}+v\right| \leqslant \sqrt{d(x)} m^{-\varepsilon_{1}}\right\} \leqslant K^{\prime} m^{-\varepsilon_{1}}
$$

с некоторой константой $K^{\prime}\left(p_{1}, p_{2}, M\right)>0$. Учитьвая, что $\left|\mathscr{A}_{x}\right| d(x) \geqslant \rho_{\ell}^{2}$ при $x \in E_{\ell}, \mathrm{c}$ помощью леммы 5 получим

$$
\mathrm{P}\left\{\left|\sum_{i=1}^{n} \xi_{i} f_{i}(x)\right| \leqslant \rho_{\ell} m^{-\varepsilon_{1}}\right\} \leqslant K^{\prime} m^{-\varepsilon_{1}} \text { для п.в. } x \in E_{\ell} \text { и всех } \ell \geqslant 1 .
$$

Далее, для каждого $\ell \geqslant n^{\varepsilon_{2}}$ определим множества

$$
\Omega_{\ell}^{\prime}:=\left\{\omega \in \Omega: \mu\left\{x \in E_{\ell}:\left|F_{n}(\omega, x)\right| \geqslant \rho_{\ell} m^{-\varepsilon_{1}}\right\} \geqslant \frac{\mu E_{\ell}}{2}\right\} .
$$

Действуя как в шаге 10, можно доказать существование события $\Omega_{0}^{\prime}$ и множества индексов $\mathscr{L}_{0}(\omega) \subset\left\{\ell: \ell \geqslant n^{\varepsilon_{2}}\right\}$ таких, что $\Omega_{0}^{\prime} \subset \bigcap_{\ell \in \mathscr{L}_{0}} \Omega_{\ell}^{\prime}, \mathrm{P}\left(\Omega_{0}^{\prime}\right) \geqslant 1-4 K^{\prime} m^{-\varepsilon_{1}}$ и

$$
\sum_{\ell \in \mathscr{L}_{0}(\omega)} \mu_{\ell} \rho_{\ell} \geqslant \frac{1}{2} \sum_{\ell \geqslant n^{\varepsilon_{2}}} \mu_{\ell} \rho_{\ell} \geqslant \frac{\sqrt{n}}{32}
$$


почти всюду на $\Omega_{0}^{\prime}$. Как в шаге 11 определим множества

$$
\Delta_{\ell}(\omega):=\left\{x \in E_{\ell}:\left|F_{n}(\omega, x)\right| \geqslant m^{-\varepsilon_{1}} \rho_{\ell}\right\}, \quad \ell \geqslant n^{\varepsilon_{2}} .
$$

По определению множеств $\Omega_{\ell}^{\prime}$ имеем $\mu \Delta_{\ell} \geqslant \mu E_{\ell} / 2 \equiv \mu_{\ell} / 2$ п.н. на $\Omega_{\ell}^{\prime}$, поэтому существуют подмножества $\Delta_{\ell}^{\prime}(\omega) \subset \Delta_{\ell}(\omega)$ такие, что $\mu \Delta_{\ell}^{\prime}=\mu_{\ell} / 2$. Положим $E^{\prime}:=\bigcup_{\ell \in \mathscr{L}_{0}} \Delta_{\ell}^{\prime}(\omega)$, для $\|\cdot\|_{m}^{*}$-нормы случайного полинома $(1)($ см. $(10))$ п.н. на $\Omega_{0}^{\prime}$ получим

$$
\begin{aligned}
\left\|F_{n}(\omega, \cdot)\right\|_{m}^{*} & \geqslant \frac{1}{\mu E^{\prime}(\omega)}\left(\int_{E^{\prime}(\omega)}\left|F_{n}(\omega, x)\right| d \mu(x)\right)\left(1-\left(1-\mu E^{\prime}(\omega)\right)^{m}\right) \\
& \geqslant \frac{2}{\sum_{\ell \in \mathscr{L}_{0}(\omega)} \mu_{\ell}}\left(\sum_{\ell \in \mathscr{L}_{0}(\omega)} \frac{\mu_{\ell}}{2} \rho_{\ell} m^{-\varepsilon_{1}}\right)\left(1-\left(1-\sum_{\ell \in \mathscr{L}_{0}(\omega)} \frac{\mu_{\ell}}{2}\right)^{m}\right) \\
& =m^{-\varepsilon_{1}}\left(\sum_{\ell \in \mathscr{L}_{0}(\omega)} \mu_{\ell} \rho_{\ell}\right) \cdot \frac{1-(1-t)^{m}}{2 t},
\end{aligned}
$$

где

$$
t=\sum_{\ell \in \mathscr{L}_{0}(\omega)} \frac{\mu_{\ell}}{2}<M n^{p_{2}} 2^{-n^{\varepsilon_{2}}}
$$

(см. (30)). Заметим, что $(1-y)^{m} \leqslant 1-m y / 2$ при $y \in(0,1 / m)$, поэтому при достаточно больших $n>n_{0}\left(p_{1}, p_{2}, M\right)$ имеем $t^{-1}\left(1-(1-t)^{m}\right)>m / 2$. Следовательно, с учетом (42) и теоремы 2 , получим

$$
\left\|F_{n}(\omega, \cdot)\right\|_{m, \infty}>128^{-1} m^{1-\varepsilon_{1}} \sqrt{n} \quad \text { п.н. на } \Omega_{0}^{\prime} .
$$

Теорема $1^{\prime}$ доказана.

ЗАМЕЧАНИЕ 4 . В условии теоремы 1 условие равномерной ограниченности третьих моментов $\mathrm{E}\left|\xi_{i}\right|^{3} \leqslant M^{3}$ можно ослабить до $\mathrm{E}\left|\xi_{i}\right|^{2+\varepsilon} \leqslant M$ с некоторым $\varepsilon>0$. При этом константы в неравенствах $(7),(8)$ будут зависеть также от $\varepsilon$. Чтобы доказать соответствуюшее утверждение, достаточно вместо предложения 1 использовать более тонкую версию центральной предельной теоремы (следствие 18.3 из [23]).

\section{4. Приложения и нерешенные проблемы}

4.1. Приложения интегрально-равномерной нормы. В [17] МонтгомериСмит и Семенов в связи с исследованием строго сингулярных вложений перестановочно-инвариантных пространств в $L_{1}[0,1]$ (т.е. пространств, нормы которых инвариантны относительно сохраняющих меру преобразований) высказали одну гипотезу, которую мы сформулируем в следующей, несколько упрощенной форме.

ГИПоТЕЗА 1. Для любого набора функиий $\left\{f_{i}\right\}_{i=1}^{n} u з L_{1}[0,1]$ maкux, что $\left\|f_{i}\right\|_{1}=1$, существует набор знаков $\left\{\theta_{i}\right\}_{i=1}^{n}, \theta_{i}= \pm 1$, для которого при всех $k=1, \ldots, n$ справедливо неравенство

$$
\left\|\sum_{i=1}^{n} \theta_{i} f_{i}\right\|_{2^{k}}^{\prime} \equiv \sup _{\substack{\Delta \subset[0,1] \\ \mu \Delta=2^{-k}}}\left\{2^{k} \int_{\Delta}\left|\sum_{i=1}^{n} \theta_{i} f_{i}(x)\right| d \mu(x)\right\} \geqslant c_{0} \sqrt{n k}
$$

(см. (11)) с некоторой абсолютной постоянной $c_{0}>0$.

С помошью теоремы 1 можно показать, что гипотеза 1 верна, по крайне мере, если параметр $k$ в неравенстве (43) принимает значения $1, \ldots,[\log n]$. Справедлива следующая 
ТЕОремА 3. Для произвольного набора $\left\{f_{i}\right\}_{i=1}^{n} \subset L_{1}[0,1]$ такого, ито $\left\|f_{i}\right\|_{1}=1$, существует последовательность знаков $\left\{\theta_{i}\right\}_{i=1}^{n}, \theta_{i}= \pm 1$, такая, что при всех $k=1, \ldots,[\log n]$ имеет место неравенство (43) с некоторой абсолютной константой $c_{0}>0$.

Доказательство теоремы 3 практически не отличается от доказательства теоремы 5 в [3], с той лиш разницей, что вместо теоремы А, сформулированной во введении, следует применить теорему 1.

Гипотеза 1 в общем виде остается открытой. Заметим, что для $k \asymp n^{\sigma}$ неравенство (43) не может быть доказано с помошью случайных знаков, поскольку во многих случаях порядок равномерной (и тем более интегрально-равномерной) нормы случайных полиномов (1) ограничен сверху величиной $\sqrt{n \log n}$, что следует, например, из оценки Салема-Зигмунда (2) (см. также теорему В из введения и теорему 3 в [25, гл. 6]).

Покажем теперь, что из оценки интегрально-равномерной нормы можно получить оценку нормы Марцинкевича. Пусть $\varphi:[0,1] \rightarrow[0,1]$ возрастающая вогнутая функция такая, что $\varphi(0)=0, \varphi(1)=1$. Тогда норма в пространстве Марцинкевича функций на отрезке [0, 1] определяется следующим образом (см., например, [17]):

$$
\|f\|_{M(\varphi)}:=\sup _{0<t<1}\left\{\frac{1}{\varphi(t)} \int_{0}^{t} f^{*}(s) d s\right\}
$$

где $f^{*}$ - неубывающая перестановка функции $f$, определяемая равенством

$$
f^{*}(s):=\inf \{\tau>0: \mu\{x \in[0,1]: f(x) \geqslant \tau\}<s\} .
$$

Как непосредственное следствие оценки (6) или (8) и эквивалентности норм $\|\cdot\|_{m}^{\prime}$ и $\|\cdot\|_{m, \infty}$ (см. теорему 2) может быть получена следующая

ТЕорема 4. Пусть для случайного полинома $F_{n}(\omega, x)$ вида (1) nри $m \leqslant n$ справедлива оченка (8), а $\varphi:[0,1] \rightarrow[0,1]$ возрастающая вогнутая функиия такая, что $\varphi(0)=0, \varphi(1)=1$. Тогда для нормы Мариинкевича полинома $F_{n}(\cdot, x)$ справедлива оценка

$$
\mathrm{E}\left\|F_{n}(\omega, x)\right\|_{M(\varphi)} \geqslant A \sqrt{n} \max _{m=2, \ldots, n}\left\{\frac{\sqrt{\log m}}{m \varphi(1 / m)}\right\}
$$

c некоторой константой $A>0$.

\section{2. О возможных обобщениях теоремы 1.}

ГИПотЕЗА 2. Теорема 1 остается верной и для случайных полиномов вида (1) по системам функиий $\left\{f_{i}\right\}_{1}^{n}$, которые удовлетворяют условию (d) (см. введение) с параметром $p \in[0,1 / 2)$ (в теореме 1 предполагается, что $p<1 / 12$ ).

Гипотезу 2 в несколько ослабленной форме можно было бы доказать, если бы мы смогли доказать утверждение следующего типа.

ГИПоТЕЗА 3. Для систем функиий $\left\{f_{i}\right\}_{1}^{n}$, которые удовлетворяют условию (d) с параметром $p \in[0,1 / 2)$ существуют константы $\varepsilon_{0} \in(0,1 / 2), L>0$ такие, что для всех $\varepsilon \in\left(0, \varepsilon_{0}\right)$, выполнено неравенство

$$
\int_{X} \sum_{1 \leqslant k \leqslant n^{1 / 2+\varepsilon}} f_{k}^{*}(x) d \mu(x) \leqslant \text { const } \cdot n^{p+L \varepsilon},
$$

здесь $f_{k}^{*}(x)$ обозначают упорядоченные по убыванию величины $\left|f_{j}(x)\right|$ в фиксированной точке $x \in X$. 
С помощью утверждения гипотезы 3 можно было бы более эффективно провести оценки в шаге 4 доказательства теоремы 1'. Именно “оптимизация" шага 4 наиболее перспективна с точки зрения обобщения теоремы 1 в сторону гипотезы 2 .

В [16] сформулирована гипотеза относительно возможности обобщения теоремы 1 на полиномы вида $\sum_{k} a_{k} \xi_{k} f_{k}$.

\section{СПИСОК ЦИТИРОВАННОЙ ЛИТЕРАТУРЫ}

[1] Salem R., Zygmund A. Some properties of trigonometric series whose terms have random signs // Acta Math. 1954. V. 91. P. 245-301.

[2] Кашин Б. С., Саакян А. А. Ортогональные ряды. М.: АФЦ, 1999.

[3] Grigoriev P. G. Estimates for norms of random polynomials // East J. Approx. 2001. V. 7. № 4. P. 445-469.

[4] Ledoux M., Talagrand M. Probability in Banach Spaces. Berlin: Springer-Verlag, 1991.

[5] Marcus M., Pisier G. Random Fourier Series with Applications to Harmonic Analysis. Princeton: Princeton Univ. Press, 1981.

[6] Dudley R. M. The series of compact subsets of Hilbert space and continuity of Gaussian processes // J. Funct. Analysis. 1967. V. 1. № 3. P. 290-330.

[7] Судаков В. Н. Меры Гаусса, Коши и є-энтропия // Докл. АН СССР. 1969. Т. 185. №1. C. $43-45$.

[8] Судаков В.Н. Гауссовские случайные процессы и меры телесных углов в гильбертовом пространстве // Докл. АН СССР. 1971. Т. 197. № 1. С. 51-53.

[9] Судаков В. Н. Геометрические проблемы теории бесконечных вероятностных распределений // Тр. МИАН. 1976. Т. 141. С. 3-190.

[10] Fernique X. Régularité des trajectoires des fonctions aléatoires gaussiennes. Berlin: SpringerVerlag, 1975. (Lecture Notes in Math. V. 480.)

[11] Slepian D. The one-sided barrier problem for Gaussian noise // Bell. System Tech. J. 1962. V. 41. P. 463-501.

[12] Kashin B., Tzafriri L. Lower estimates for the supremum of some random processes // East J. Approx. 1995. V. 1. №1. P. 125-139.

[13] Kashin B., Tzafriri L. Lower estimates for the supremum of some random processes, II // East J. Approx. 1995. V. 1. № 3. P. 373-377.

[14] Kashin B., Tzafriri L. Lower estimates for the supremum of some random processes, II // Preprint № 95-85. Bonn: Max-Plank Institut für Mathematik, 1995.

[15] Григорьев П. Г. Оценки норм случайных полиномов и их приложение // Матем. заметки. 2001. T. 69. №6. С. 950-954.

[16] Григорьев П. Г. Случайные и специальные полиномы по общим функциональным системам // Дисс. ... канд. физ.-матем. наук. М.: МИАН, 2002.

[17] Montgomery-Smith S., Semenov E. M. Embeddings of rearrangement invariant spaces that are not strictly singular // Positivity. 2000. V. 4. P. 397-402.

[18] Chang K. L., Erdös P. On the application of the Borel-Cantelli lemma // Trans. Amer. Math. Soc. 1952. V. 72. P. 179-186.

[19] Спринджук В. Г. Метрическая теория диофантовых приближений. М.: Наука, 1977.

[20] Šidak Z. Rectangular confidence regions for the means of multivariate normal distributions // J. Amer. Statistic Assoc. 1967. V. 62. № 318. P. 626-633.

[21] Šidak Z. On multivariate normal probabilities of rectangles // Ann. Math. Statistic. 1968. V. 39. P. 1425-1434.

[22] Ротарь В. И. Неравномерная оценка скорости сходимости в многомерной центральной предельной теореме // Теория вероятн. и ее примен. 1970. Т. 15. № 4. С. 647-665.

[23] Бхаттачария Р. Н., Р анго Рао Р. Аппроксимация нормальным распределением и асимптотические разложения. М.: Наука, 1982.

[24] Ширяев А. Н. Вероятность. М.: Наука, 1989.

[25] Кахан ЖК.-П. Случайные функциональные ряды. М.: Мир, 1973.

Московский государственный

Поступило

университет им. М.В. Ломоносова

11.04 .2002

E-mail: pgrigoriev@yahoo.com

Исправленный вариант

06.12 .2002 